\title{
LA FORMATION ET L'ÉVOLUTION DE LA BOUCLE CHEZ LE FETUS ET L'AGNEAU KARAKUL LES FACTEURS DE QUALITÉ DE LA FOURRURE ET LEUR IMPORTANCE POUR LA SÉLECTION \\ (DEUXIÈM PARTIE)
}

PAR

\section{NACER KECHAWARZ}

\section{CHAPITRE IV}

\section{LA QUALITÉ DE LA FOURRURE}

Outre la fermeté et le dessin des boucles et l'épaisseur réduite de la peau que nous avons vus dans les précédents chapitres, le lustre du pelage et les opérations de tannage interviennent dans la qualité de la fourrure. Tandis que le lustre n'a pas été étudié, les facteurs technologiques ont fait 1'objet de quelques observations.

\section{A. - LE LUSTRE}

Le lustre est un caractère très recherché dans l'appréciation de la fourrure. Il est déterminé par la proportion de lumière réfléchie et de lumière diffusée. Par conséquent il varie avec l'angle d'incidence de la lumière et la direction de l'observation.

Le lustre varie d'un agneatı à l'autre et pour le même individu, il varie avec 1'âge.

\section{Facteurs du lustre.}

Les principaux facteurs susceptibles de jouer un rôle dans le degré du lustre de la fourrure d'agneau Karakul seraient la composition du liquide amniotique, la forme et les dimensions et l'intégrité des écailles de la cuticule, et la disposition des poils.

Le liquide amniotique ne saurait être considéré comme un facteur du lustre puisque celui-ci est conservé même après lavage et nettoyage des peaux au cours de l'apprêt.

L'examen des empreintes des écailles sur la gélatine nous a montré 
que non seulement il n'y a pas de différence essentielle de forme des écailles entre les peaux plus ou moins brillantes, mais que pour le même diamètre du poil, les écailles chez la race Karakul ressemblent à celles des poils d'autres races ovines.

I,e facteur le plus important est sa11s doute la disposition et l'uniformité des poils. Une peau est d'autant plus brillante qu'elle offre une plus grande surface lisse à la lumière ; c'est pourquoi les peaux dont les poils sont couchés à plat et à peine bouclés (Galyac et Breitschwanz) sont les plus brillantes. Pour la même raison une peau à boucles plates et larges est plus lustrée qu'une peau à boucles élevées.

Une peau Galyac ne comporte pas de dessin en relief. Cependant, certaines peaux Galyac présentent un dessin lumineux qui se modifie complètement quand l'angle d'incidence vatie. Ceci est dù à l'existence des zones où l'orientation des poils est différente. Ainsi sur une même peau Galyac de couleur noire uniforme, on peut observer, suivant l'incidence de la lumière, des zones à lustre noir, argenté, métallique ou bleuté.

\section{Mesure du lustre.}

Dans la pratique l'estimation du lustre se fait à l'œil. Cette méthode est assez subjective et varie avec l'observateur, avec l'intensité lumineuse et surtout avec l'incidence de la lumière. On pourrait done se demander s'il n'y a pas un autre moyen objectif pour mesurer le lustre dans des conditions plus précises et constantes. C'est ce que nous avons essayé de réaliser afin de classer les peaux d'après leurs lustres.

Le lustre ou le brillant peuvent être définis comme étant le rapport de la luminance mesurée à $45^{\circ}$ à celle mesurée suivant la normale.

La luminance dans une direction donnée d'une surface énettant de la lumière est le quotient de l'intensité lunineuse mesurée dans cette direction, par l'aire projetée de cette surface sur un plan perpendiculaire à la direction considérée.

L : Luminance ;

$\mathrm{J}$ : Intensité lumineuse ;

₹ : L’aire éclairée.

Nous avons décrit par ailleurs les méthodes de mesure du lustre : nitomètre et mesure photoélectrique.

\section{a) Méthode du nitomètre}

Les mesures ont été effectuées sur deux lots de peaux. A l'intérieur de chaque lot, les peaux ont été classées d'après l'estimation à l'œil, de leur lustre et numérotées par ordre décroissant de ce lustre.

Le tableau XVI montre les valeurs obtenues pour le premier lot. La 
peau $\mathrm{n}^{\circ}$ I étant la plus brillante à l'œil, réfléchit une grande partie de la lumière tandis que la peau $\mathrm{n}^{\circ} 3$ qui est moins brillante diffuse davantage la lumière.

\section{TABLEAU XVI}

Lot I : Concordance de la classification du lustre à l'ail et du rapport :

$$
R=\frac{\text { observation à } 45^{\circ}}{\text { observation à } 90^{\circ}} \text {. }
$$

\begin{tabular}{c|c|c|c|c}
\hline \hline \multirow{2}{*}{$\begin{array}{c}\text { Numéros } \\
\text { de peau }\end{array}$} & $\begin{array}{c}\text { Angle incident } \\
\text { d'éclairement }\end{array}$ & \multicolumn{2}{|c|}{ Valeurs indiquées par le tambour } & \multirow{2}{*}{$\mathrm{R}$} \\
\cline { 3 - 5 } & & Observation à $45^{\circ}$ & Observation à $90^{\circ}$ & \\
\hline $\mathrm{I}$ & $45^{\circ}$ & 32 & $\mathrm{14}$ & 2,25 \\
2 & $45^{\circ}$ & 36 & 17 & $2, \mathrm{I}$ \\
3 & $45^{\circ}$ & 30 & $\mathrm{I} 7$ & $\mathrm{I}, 8$ \\
\hline \hline
\end{tabular}

TABLEAU XVII

Lot 2 : Concordance de la classification du lustre à l'cil et durapport $R$.

\begin{tabular}{|c|c|c|c|c|}
\hline \multirow{2}{*}{$\begin{array}{l}\text { Numéro } \\
\text { Peau }\end{array}$} & \multirow{2}{*}{$\begin{array}{l}\text { Angle incident } \\
\text { d'éclairement }\end{array}$} & \multicolumn{2}{|c|}{ Valeurs indiquées par le tambour } & \multirow{2}{*}{$\mathrm{R}$} \\
\hline & & Observation à $45^{\circ}$ & Observation à $90^{\circ}$ & \\
\hline $1 \ldots \ldots \ldots$ & $45^{\circ}$ & 32,2 & II, 6 & 2,86 \\
\hline $2 \ldots \ldots \ldots$ & $45^{\circ}$ & 21,4 & I 2,8 & $\mathrm{I}, 67$ \\
\hline $3 \ldots \ldots \ldots$ & $45^{\circ}$ & 23,0 & 15,2 & $\mathrm{r}, 5 \mathrm{I}$ \\
\hline $4 \ldots \ldots \ldots$ & $45^{\circ}$ & 29,0 & 14,9 & 1,48 \\
\hline $5 \ldots \ldots \ldots$ & $45^{\circ}$ & I9, O & II, 8 & $\mathrm{r}, 6 \mathrm{I}$ \\
\hline $6 \ldots \ldots \ldots$ & $45^{\circ}$ & 19,1 & I 3,4 & $\mathbf{x}, 45$ \\
\hline
\end{tabular}

Les peaux du second lot ont été expérimentées avec une intensité lumineuse plus faible. I es tableaux XVI et XVII montrent que les

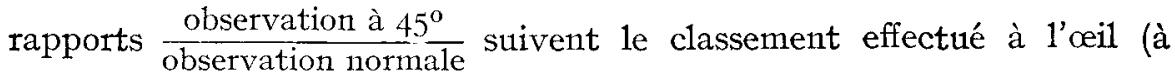
l'exception du $\mathrm{n}^{0} 5 \mathrm{du}$ second lot). Mais l'écart entre les différentes peaux est assez faible. I1 y a une insuffisance de sensibilité, 1'appareil n'ayant pas été conçu pour mesurer des luminances si rapprochées. D'autre part, les deux plages lumineuses étant hétérochromes, leur égalisation est assez difficile car la plage correspondante à l'échantillon est grise alors que celle de la lampe de référence est jaunâtre.

Pour arriver à faire des mesures convenables il faudrait avoir un dispositif de gradation de lumière de référence plus sensible. En d'autres termes, le coin photométrique du nitomètre devrait être de densité optique plus faible pour une même rotation du tambour. Des mesures précises exigeraient aussi des plages homochromes.

Le rapport $\frac{\text { observation à } 45^{\circ}}{\text { observation normale }}$ est en principe indépendant de la couleur de l'échantillon et dépend uniquement du brillant. Ainsi par 
exemple pour une peau marron dont le brillant à l'oil est voisin de celui de la peau noire $1^{\circ} 3 \mathrm{du}$ premier lot, nous avons trouvé $\mathrm{R}=43 / 24=\mathrm{I}, 79$ qui est très près du $I, 8$ trouvé pour ladite peau $n^{\circ} 3$.

\section{b) Méthode photoélectrique}

Cette méthode paraît séduisante mais après quelques essais nous nous sommes aperçus qu'elle comporte certains inconvénients notamment le manque de sensibilité de la cellule du fait du flux lumineux trop faible. Deux remèdes sont préconisés : ou bien augmenter la sensibilité de la cellule en utilisant un dispositif amplificateur, difficile à utiliser, ou bien augmenter l'intensité de la source tout en gardant la même lentille qui fournit une image de $2 \mathrm{~mm}$ de diamètre. Mais on a intérêt à ce que l'image qui tombe sur la cellule soit de l'ordre de grandeur de la photocathode pour utiliser toute la surface sensible de la cellule. La méthode photoélectrique manque de fidélité du fait de la variation de sensibilité de la cellule avec différents facteurs tels que le temps d'exposition à la lumière, la température, etc.

\section{CONCLUSION}

Le lustre de la fourrure peut être considéré comme étant le rapport de la luminance mesurée à $45^{\circ}$ à celle mesurée suivant la normale, le rayon incident formant dans les deux cas un angle de $45^{\circ}$.

Les mesures effectuées par la méthode photoélectrique n'ont pas donné satisfaction. Par contre les résultats obtenus par la méthode du nitomètre concordent dans l'ensemble avec l'estimation visuelle. Nos résultats manquent de précision car l'appareil utilisé n'était pas conçu pour mesurer des valeurs de luminance aussi rapprochées. Nous avons suggéré les perfectionnements à apporter.

Mais le reproche essentiel que 1'on peut faire à ces deux méthodes c'est qu'elles donnent la moyenne de points plus ou moins brillants alors que l'œil perçoit les nuances, entre chacun de ces points, qui donnent l'aspect séduisant à la fourrure. Compte tenu de cette réserve nous pensons qu'un nitomètre modifié pourrait servir à standardiser l'appréciation du lustre de la fourrure.

En ce qui concerne les causes du lustre, les seuls facteurs incontestables sont la disposition régulière des ipoils dans la boucle formant une surface lisse, et l'importance relative de la surface ainsi offerte à la lumière. C'est pour cela que les peaux de fotus sont plus brillantes que celles d'agneaux et les boucles larges ont plus d'éclat que les petites boucles. Les dimensions des écailles de la cuticule n'interviennent pas directement puisque pour le même diamètre du poil, ces écailles ont, chez le karakul, 
à peu près les mêmes formes et dimensions que chez les autres races dont les agneaux ne possèdent pas de pelage brillant.

\section{B. - LE TANNAGE}

\section{HISTORIQUE}

Les peaux d'agneau Karakul subissent des modifications durant les traitements industriels ayant pour but de les rendre utilisables à la pelleterie. Ces modifications sont à la fois quantitatives et qualitatives. FröLICH, HeRre et HorNitscheK (I938) trouvent au cours du tannage une réduction de 4 à $2 \mathrm{I} \mathrm{p}$. Ioo en poids; le poids des peaux brutes étant de I $4 \mathrm{I}-349 \mathrm{~g}$ pour la race pure et I85-377 $\mathrm{g}$ pour les agneaux croisés. La superficie des peaux tannées se réduit, selon FröLICn et HorNITSCHEK (I94I), de $I_{4}$ à 20 p. Ioo par rapport à l'état frais.

Quant aux modifications de la qualité des peaux durant les opérations de tannage, certains auteurs pensent avec THompson (I938) que les boucles orientées vers la queue se déroulent au cours du tannage; Kononova (I937) trouve que, durant cette opération, la plupart des boucles s'orientent vers la tête. Mais Judis (I933) a prouvé, par ses photographies, l'identité de structure et de qualité des peaux à l'état frais et à l'état apprêté.

Les types de boucle susceptibles de s'améliorer au cours du tannage seraient les types anneau et semi-anneau qui donneraient naissance au type haricot (Kononova, I937).

Par contre, seraient susceptibles de s'ouvrir le type de boucle étendue (en cote) orienté rers la queue, et le type en tube très développé et à poils longs.

\section{RÉSULTATS DE RECHERCHES}

Les peaux d'agneaux Karakul, depuis la production jusqu'à la confection, passent par trois stades:

- État frais : peaux fraîchement décharnées aussitôt après 1'abattage de l'agneau.

- État brut : peaux simplement séchées ou mises en confit et séchées afin d'éviter la détérioration en attendant le tannage.

- État tanné : peaux tannées et apprêtées, souvent même teintes et lustrées de façon définitive.

Les peaux d'agneaux rencontrées sur le !marché sont de nature et de provenance diverses; aussi avons-nous envisagé de les étudier séparément. En effet, bien qu'elles appartiennent aux agneaux de la race Karakul, elles proviennent d'individus ou de groupes d'individus génétique- 
ment différents, notamment en ce qui concerne les croisements. Par ailleurs, les traitements subis par les peaux fraîches ne sont pas les mêmes et varient suivant la provenance de celles-ci. C'est ainsi que les peaux afghanes et russes, provenant de la même zone géographique (les deux rives de 1'Amou-Daria) et subissant les mêmes traitements, se ressemblent davantage. Les peaux originaires de 1'Afrique du Sud-Ouest (S. W. A.) sont très différentes des précédentes par leurs formes et par les traitements qu'elles subissent. En effet, elles ne sont pas mises en confit mais seulement séchées après avoir été fortement étirées, ce qui augmente leur superficie à l'état brut.

Les peaux produites au Centre National de Recherches Zootechniques (C. N. R. Z.) ont été traitées de façon très différente des traitements commerciaux. C'est pour cette raison que nous les étudierons séparément pour bien mettre en évidence l'action de ces traitements spéciaux.

Les modifications que subissent les peaux Karakul au cours des traitements industriels portent d'une part sur les dimensions (longueur, largeur, surface, épaisseur) et d'autre part sur l'aspect de la fourrure. Les variations des dimensions se répercutent naturellement sur le poids de la peau.

Nos recherches sur les peaux commerciales sont limitées aux états brut et tanné. L'état frais est étudié sur les peaux produites au Centre National de Recherches Zootechniques (C. N. R. Z.).

\section{Peaux commerciales.}

\section{a) Modification de la surface}

a) Peaux afghanes: Les peaux que nous avons étudiées (fig. 64) mesuraient en moyenne $2 \mathrm{I}, 25 \mathrm{dm}^{2}$ de surface à l'état brut et $23,30 \mathrm{dm}^{2}$

\section{TABLEAU XVIII}

Modification de la surface des peaux Karakul afghanes (type Tchakmaki) au cours du tannage (mesurée en $d^{2}{ }^{2}$ )

\begin{tabular}{|c|c|c|c|c|}
\hline \multirow{2}{*}{ Numéro de peau } & \multirow{2}{*}{ Brute } & \multirow{2}{*}{ Tannée } & \multicolumn{2}{|c|}{ Augmentation } \\
\hline & & & en $d m^{2}$ & $\%$ \\
\hline I $\ldots \ldots \ldots \ldots \ldots$ & 21,75 & 24,50 & 2,75 & $\simeq 2,64$ \\
\hline $2 \ldots \ldots \ldots \ldots$ & $2 \mathrm{I}, 75$ & 24,12 & 2,37 & 10,89 \\
\hline $3 \ldots \ldots \ldots \ldots \ldots$ & 20,00 & 21,50 & $\mathrm{r}, 5^{\circ}$ & 7,00 \\
\hline $4 \ldots \ldots \ldots \ldots \ldots$ & I 9,50 & 20,00 & $0,5 \circ$ & 2,56 \\
\hline & $25,5^{\circ}$ & $26,7,5$ & $I, 25$ & 4,90 \\
\hline $6 \ldots \ldots \ldots \ldots$ & 17,75 & 20,00 & 2,25 & $\begin{array}{r}12,67 \\
\end{array}$ \\
\hline $7 \ldots \ldots \ldots \ldots \ldots$ & $2 \mathrm{I}, 25$ & 24,37 & 3,12 & {$[4,68$} \\
\hline $8 \ldots \ldots \ldots \ldots \ldots$ & $19,5^{\circ}$ & $21,5^{\circ}$ & 2,00 & 10,26 \\
\hline $9 \ldots \ldots \ldots \ldots \ldots$ & 24,25 & 27,00 & 2,75 & 11,34 \\
\hline Moyenne .......... & 21,25 & $2,3,30$ & 2,05 & 9,66 \\
\hline
\end{tabular}


à l'état tanné. Elles subissent donc une augmentation de 2,05 $\mathrm{dm}^{2}$ à la suite du tannage, soit 9,66 p. Ioo. I,e tableau XVIII montre les différences individuelles entre les diverses peaux.

L'augmentation de la surface n'est pas la même sur les différentes parties du corps. Nous l'avons mis en évidence grâce aux mensurations des segments $\mathrm{AB}, \mathrm{CD}, \mathrm{IIF}, \mathrm{GH}, \mathrm{IJ}$ et $\mathrm{KI}$, choisis sur la peau pour donner

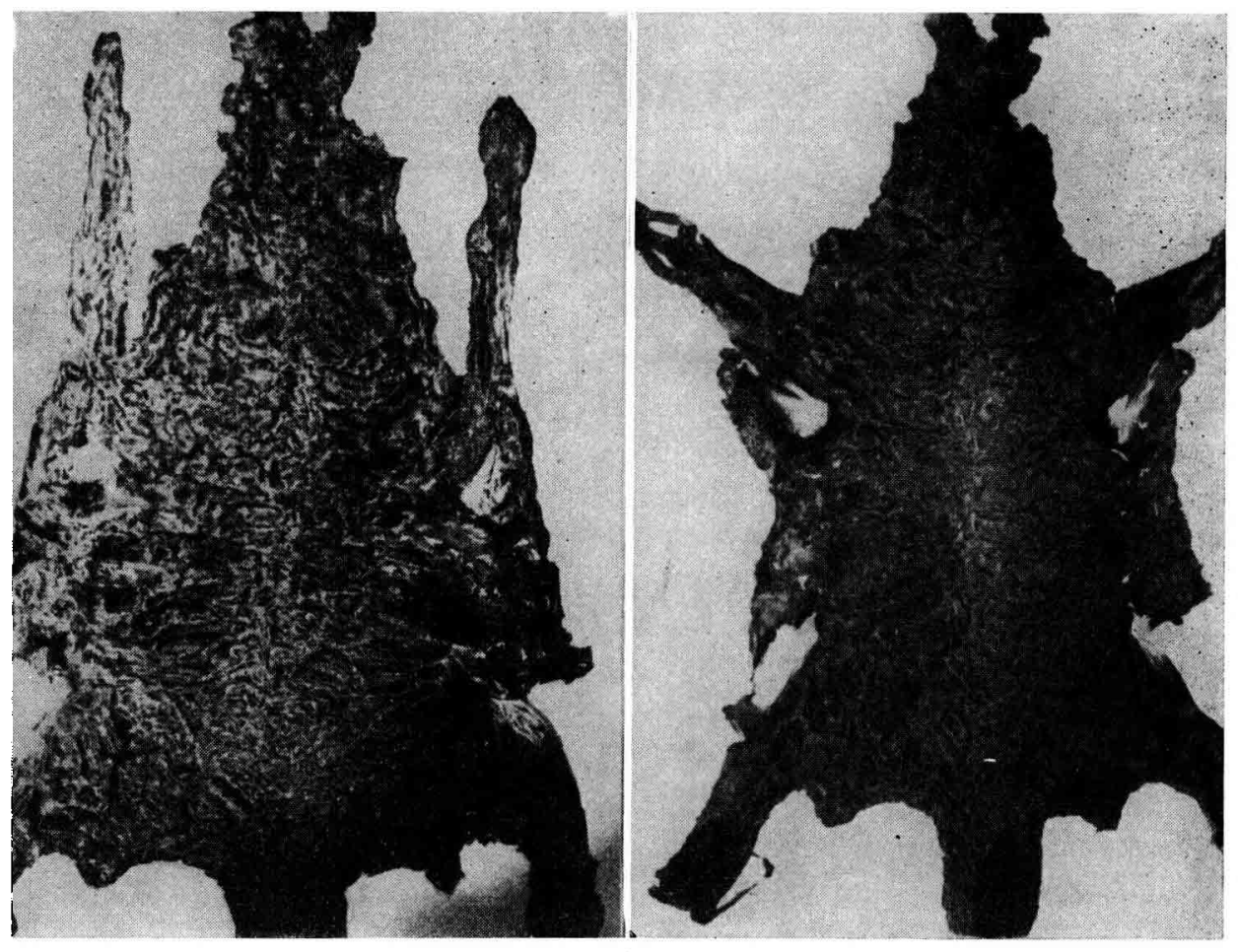

FIG. 6.4. - Peau Karakul du type "Tchakmaki ", avant et après tannage. Remarquer la forme d'ensemble et la régularité du dessin des boucles, olitenues à la suite du tannage (á droite).

une idée sur la largeur et la longueur de la peau. Ces segments ont été choisis de la manière suivante:

$\mathrm{AB}:$ Distance de l'extrémité de la tête à celle de la queue.

CI) : Distance entre 1'aisselle et l'aine.

EF : Largeur de la peau au niveau de l'épaule.

$\mathrm{GH}$ : Distance entre les aisselles des membres antérieurs.

IJ : Largeur de la peau au niveau de la croupe.

$\mathrm{KL}_{4}$ : Largeur de la peau au niveau du milieu du corps.

Le tableau XIX montre que la longueur AB mesurée de la tête à la queue ne varie pas après le tannage ou à peine ( $\mathrm{I}$ p. IOo). Par contre au niveau CD il y a une augmentation de 9 p. Ioo en longueur. Fn ce qui 
concerne la largeur, la peau s'élargit de 2 I à I5 p. Ioo respectivement aux niveaux EF et $\mathrm{GH}$ de la partie antérieure du corps mais jelle rétrécit de II p. Ioo au niveau de la taille (KI).

\section{TABleaU XIX}

Modification de la taille moyenne des peaux Karakul au cours du tannage suivant les segments $A B, C D$, etc. (Modification en $p$. Ioo de la valeur à l'état brut, mesurée en $\mathrm{cm}$ ).

\begin{tabular}{|c|c|c|c|c|c|c|c|c|c|c|c|c|}
\hline & \multicolumn{6}{|c|}{ Valeur avant tannage } & \multicolumn{6}{|c|}{ Modification après tamage $(\%)$} \\
\hline & $\Lambda B$ & $\mathrm{Cl}$ & $\mathrm{EF}$ & GII & IJ & $\mathrm{KL}$ & $A B$ & $\mathrm{CD}$ & $\mathrm{EF}$ & $\mathrm{GH}$ & IJ & $\mathrm{KI}$ \\
\hline l'eaux afghanes & 86,88 & 35,26 & 33,88 & 22,55 & 44,00 & 40,05 & $-I$ & +9 & $+2 \mathbf{I}$ & $-1+15$ & $-0,25$ & - I I \\
\hline $\begin{array}{l}\text { Peaux sud-afri- } \\
\text { raines......... }\end{array}$ & 71,88 & 44,33 & 43,44 & 24,55 & $44, \infty 0$ & 43,88 & $\div \quad 0,1$ & -9 & -7 & $|+17|$ & - I I & - 18 \\
\hline
\end{tabular}

3) Peaux russes: Les peaux russes subissent également une augmentation de surface de l'ordre de $8 \mathrm{p}$. Ioo. Les mesures ont été faites sur trois peaux du type Rubresti tolsti qui comptaient en moyenne $23,25 \mathrm{dm}^{2}$ à l'état brut et $25, \mathrm{I} 5 \mathrm{dm}^{2}$ après tannage, soit une augmentation de $\mathrm{I}, 90 \mathrm{dm}^{2}$.

Y) Peaux sud-africaines: Les résultats des mensurations effectuées avant et après le tannage, et indiquées par le tableau $\mathrm{XX}$, montrent que les peaux sud-africaines éprouvent une réduction de surface considérable au cours du tannage. Cela tient sans doute au fait que ces peaux sont fortement étirées en tous sens, et séchées, étendues sur des cadres en bois qui les maintiennent étirées. Les peaux brutes mesurent en moyenne $26,79 \mathrm{dm}^{2}$ de surface. Une fois tannées, elles ne mesurent que $23, \mathrm{I} 7 \mathrm{dm}^{2}$, soit une réduction de $3,62 \mathrm{dm}^{2}$. Autrement dit la réduction de surface est de I3,5I p. Ioo au cours du tannage.

\section{TABLEAU XX}

Modification de la surface des peaux Karakul sud-africaines au cours du tannage (mesurée en $\mathrm{dm}^{2}$ )

\begin{tabular}{|c|c|c|c|c|}
\hline \multirow{2}{*}{$\begin{array}{l}\text { Numéro de } \\
\text { peaul }\end{array}$} & \multirow{2}{*}{ Brute } & \multirow{2}{*}{ Tanne } & \multicolumn{2}{|c|}{ Réduction } \\
\hline & & & en $d m^{2}$ & $\%$ \\
\hline $\mathbf{I} \ldots \ldots \ldots \ldots$ & 31,00 & 25,75 & 5,25 & 16,93 \\
\hline $2 \ldots \ldots \ldots \ldots$ & 26,00 & 22,12 & 3,88 & 14,92 \\
\hline $3 \ldots \ldots \ldots \ldots$ & $24,5^{\circ}$ & 21,75 & 2,75 & $I I, 22$ \\
\hline $5 \ldots \ldots \ldots \ldots$ & 26,25 & 22,12 & 4,13 & I 5,73 \\
\hline $6 \ldots \ldots \ldots \ldots$ & 24,25 & 21,60 & 2,65 & 10,92 \\
\hline $8 \ldots \ldots \ldots$ & 24,17 & 20,50 & 3,67 & $r_{5}, 20$ \\
\hline $9 \ldots \ldots \ldots \ldots$ & 30,75 & $28,5^{\circ}$ & 2,25 & $7,3 \mathrm{I}$ \\
\hline Io.. $\cdots \ldots \ldots \ldots$ & 26,57 & 23,25 & 3,32 & $12,5^{\circ}$ \\
\hline $2 I \ldots \ldots \ldots \ldots$ & 27,64 & 23,12 & 4,52 & 16,37 \\
\hline Moyenne & 26,79 & 23,17 & 3,62 & $13,5^{\mathrm{I}}$ \\
\hline
\end{tabular}


Cette réduction de surface est inégalement répartie sur la peau entière. Elle porte surtout sur la largeur au niveau de la taille et de la croupe. Quant à la longueur, elle ne varie pas sur la ligne craniocaudale, mais se réduit sur les deux flancs (tableau XIX).

\section{b) Modification de l'épaisseur}

Pour la détermination de l'épaisseur, nous avons choisi un certain nombre de points sur le côté latéral du cou, le dos, la queue et les membres. Pour les peaux produites au C. N. R. Z. nous avons également mesuré l'épaisseur de la peau sur la tête, le cou, la croupe et le ventre. I,es mesures ont été faites au Palmer.

a) Peaux afghanes: Le tableau XXI montre qu'au cours du tannage l'épaisseur de la peau se réduit à tous les niveaux mais cette réduction n'est pas la même pour les différents points où l'épaisseur a été mesurée. L'amincissement varie de 6 à 23 p. Ioo suivant les points considérés. Le minimum de variation ( $6 \mathrm{p}$. Ioo) est noté au niveau du milieu des membres, puis viennent successivement la queue (IO,4 p. IOO), le dos (I5 p. IOO) et le cou qui accuse une réduction d'épaisseur de la peau atteignant 23 p. Ioo de sa valeur à l'état brut.

Cette réduction est la conséquence de deux facteurs. D'une part une petite épaisseur du confit recouvrant la peau faisait paraître celle-ci plus épaisse qu'elle ne l'était réellement. D'autre part l'écharnage de la peau au cours du tannage lui enlève une certaine épaisseur. L'écharnage est plus poussé sur le cou car cette région est particulièrement grattée au couteau pour l'amincir. Ces deux phénomènes se justifient d'ailleurs par une forte diminution du poids (élimination du confit + écharnage).

Quant à l'épaisseur réelle de la peau, nous ne pouvons rien en dire à priori tant que nous n'avons pas éliminé ou déterminé la part qui revient au confit et à l'écharnage. La solution nous sera donnée par les peaux sud-africaines dont l'écharnage étant plus complet au moment du sacrifice des agneaux, est minime au cours du tannage. Par ailleurs les peaux sud-africaines ne sont pas mises en confit.

3) Peaux sud-africaines : Contrairement aux peaux afghanes, les peaux sud-africaines accusent une augmentation d'épaisseur au cours du tannage. Malgré l'écharnage qui est relativement faible au cours du tannage, cette augmentation d'épaisseur varie de 28 à 50 p. roo suivant les points considérés (tableau XXII). Elle est minimum (28 p. 10o) pour le cou où l'écharnage est plus poussé ; pour la queue elle est de $37 \mathrm{p}$. Ioo, pour le dos de $44 \mathrm{p}$. Ioo et pour les membres, de 50 p. roo.

Annales de Zootechnie. -- $195^{8}$. 


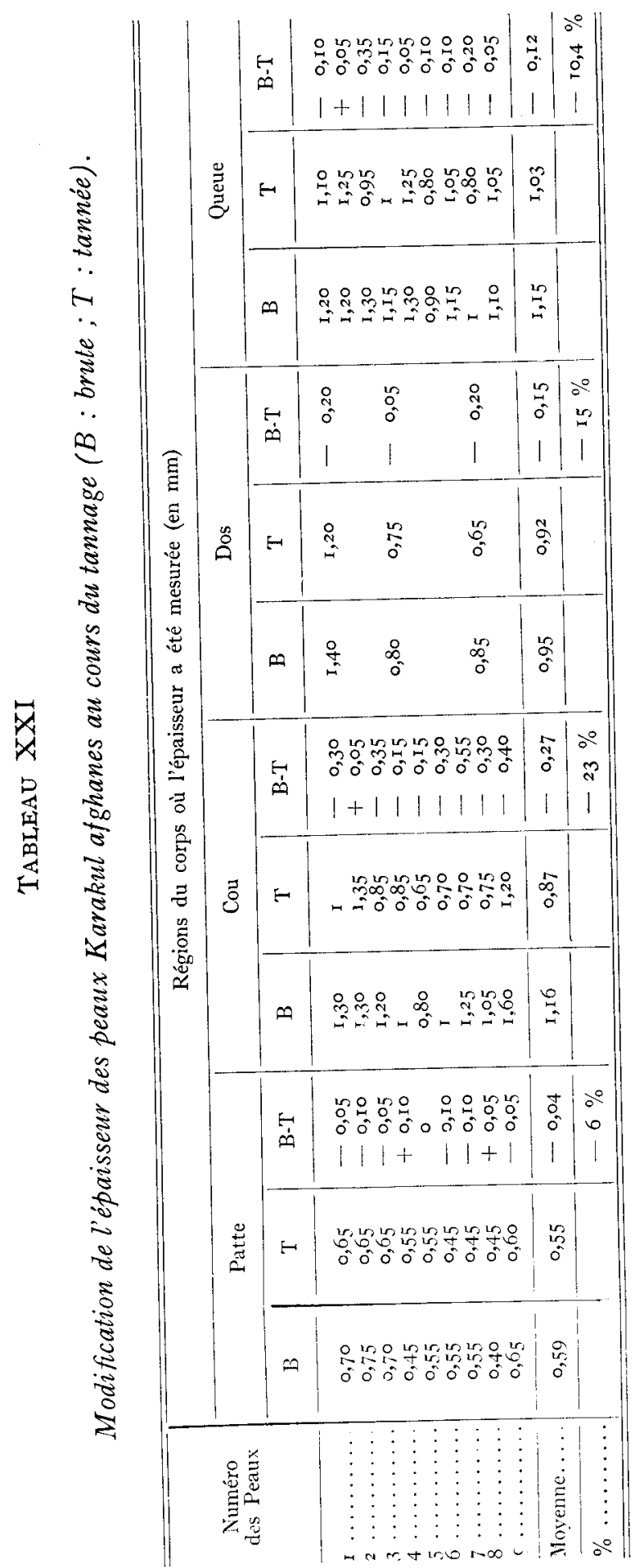




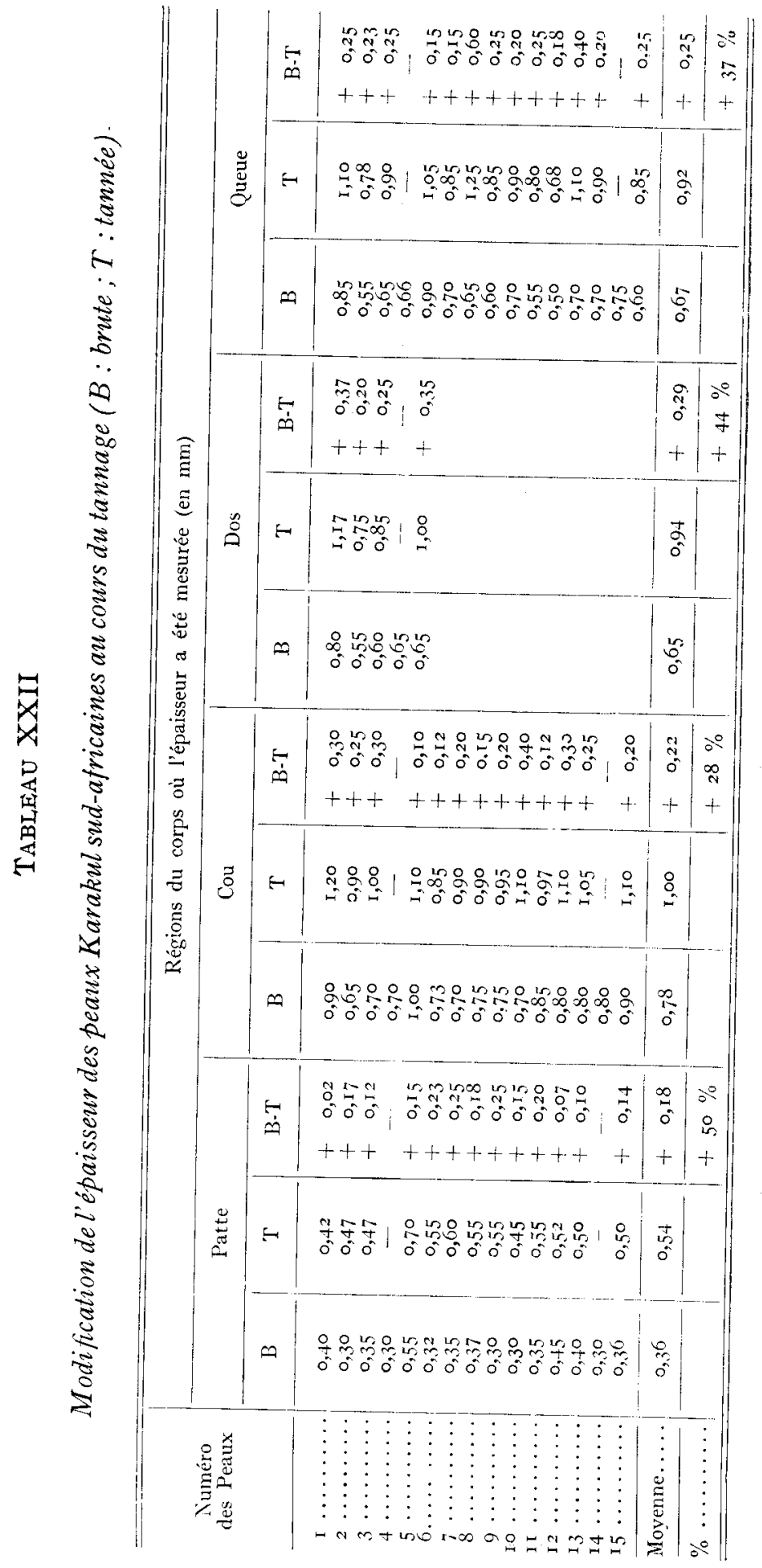




\section{c) Modification du poids}

Les expériences en vue de la détermination de la variation du poids ont été effectuées avant et après tannage sur I9 peaux afghanes, Io russes et I7 sud-africaines. I,es tableaux XXIII et XXIV donnent les résultats obtenus. Ces résultats sont récapitulés dans le tableau XXV.

\section{TABIEAU XXIII}

Modification du poids (en grammes) des peaux Karakul afghanes et sudafricaines au cours du tannage

\begin{tabular}{|c|c|c|c|c|c|c|c|c|c|}
\hline \multicolumn{5}{|c|}{ Peaux afghanes } & \multicolumn{5}{|c|}{ Peaux sud-africaines } \\
\hline \multirow{2}{*}{$N^{\circ}$ de peau } & \multirow{2}{*}{ Brute } & \multirow{2}{*}{ Tannée } & \multicolumn{2}{|c|}{ Réduction } & \multirow{2}{*}{ No de peau } & \multirow{2}{*}{ Brute } & \multirow{2}{*}{ Tannée } & \multicolumn{2}{|c|}{ Réduction } \\
\hline & & & en $g$ & $\%$ & & & & enl g & $\%$ \\
\hline$-\cdots-$ & -7 & - & -- & - & $--\cdots-\cdots--$ & - & $\cdots \cdot-\cdots$ & $-\ldots-$ & $\ldots$ \\
\hline $\mathbf{I} \ldots \ldots \ldots$ & $3^{6} 3$ & 252 & I I I & 30,50 & $2 \ldots \ldots \ldots$ & 187 & 175 & 12 & 6,42 \\
\hline $2 \ldots \ldots \ldots$ & 338 & 220 & rog & 32,24 & $3 \ldots \ldots \ldots$ & 202 & 106 & 6 & 2,97 \\
\hline $3 \ldots \ldots \ldots$ & 298 & 204 & ci.t & 31,54 & $5 \ldots \ldots \ldots$ & 2.39 & 210 & 20 & 8,37 \\
\hline $4 \ldots \ldots \ldots$ & 24.3 & I 190 & 5.3 & $2 \mathrm{I}, \mathrm{SI}$ & $7 \ldots \ldots \ldots$ & 20,3 & 183 & 20 & 9,85 \\
\hline $5 \ldots \ldots \ldots$ & 355 & 225 & ijo & $2 S, 5 \bar{T}$ & $s \ldots \ldots \ldots$ & 180 & 166 & 14 & 7,78 \\
\hline $6 \ldots \ldots \ldots$ & 25.3 & 1.35 & -8 & $3^{(1), 62}$ & 9. $\ldots \ldots \ldots$ & 2.35 & 2.33 & 2 & 0,85 \\
\hline $7 \ldots \ldots \ldots$ & 352 & $21+$ & is 8 & $31,+1$ & $10 \ldots \ldots \ldots$ & 192 & 185 & 7 & 3,64 \\
\hline $8 \ldots \ldots \ldots$ & 230 & 17 & $\therefore$ & 25065 & $1+\ldots \ldots \ldots$ & 252 & 244 & 8 & 3,17 \\
\hline $9 \ldots \ldots \ldots$ & 44.3 & 300 & $1+i$ & 32,28 & $\mathrm{I} 2 \ldots \ldots \ldots$ & 226 & 218 & 8 & 3,54 \\
\hline $51 \ldots \ldots \ldots$ & +10 & 30.5 & 105 & 25,60 & $13 \ldots \ldots \ldots$ & 23,32 & 226 & 6 & $2,5^{8}$ \\
\hline $5_{2}^{2} \ldots \ldots \ldots$ & .365 & 210 & 125 & $3+, 25$ & $15 \ldots \ldots \ldots$ & 208 & 197 & I I & 5,28 \\
\hline 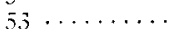 & 395 & 300 & 95 & $2+, 05$ & $i t \ldots \ldots \ldots$ & 2.30 & 219 & I I & 4,78 \\
\hline $54 \ldots \ldots \ldots$ & $27^{6}$ & 215 & (i) & 22,10 & $17 \ldots \ldots \ldots$ & 298 & 284 & 14 & 4,70 \\
\hline $55 \ldots \ldots \ldots$ & ito & 255 & 85 & 25,00 & Is $\ldots \ldots \ldots$ & 185 & 369 & 16 & 8,65 \\
\hline $56 \ldots \ldots \ldots$ & 300 & 225 & .5 & 25.00 & I $9 \ldots \ldots \ldots$ & $2+4.3$ & 224 & J9 & 7,82 \\
\hline $57 \ldots \ldots \ldots$ & $33^{\circ}$ & 25.5 & 75 & $22,7,3$ & $20 \ldots \ldots \ldots \ldots$ & $2 \mathrm{I}_{3}$ & $2 \mathrm{II}$ & 2 & 0,94 \\
\hline $58 \ldots \ldots \ldots$ & 355 & 275 & So & $22,5,3$ & $2 \mathrm{I} \ldots \ldots \ldots$ & 185 & I $8_{2}$ & 3 & 1,62 \\
\hline $59 \ldots \ldots \ldots$ & $35^{\circ}$ & 20,5 & 85 & 24,28 & & & & & \\
\hline $60 \ldots \ldots \ldots$ & 305 & 2.35 & $70 ̈$ & 22,95 & & & & & \\
\hline Moyenne.... & 324.30 & 235,15 & 80,15 & 27,42 & & 218,23 & $207,7 \circ$ & 10,53 & 4,80 \\
\hline
\end{tabular}

\section{Tableau XXIV}

Modification du poids des peaux Karakul russes au cours du tannage (en grammes)

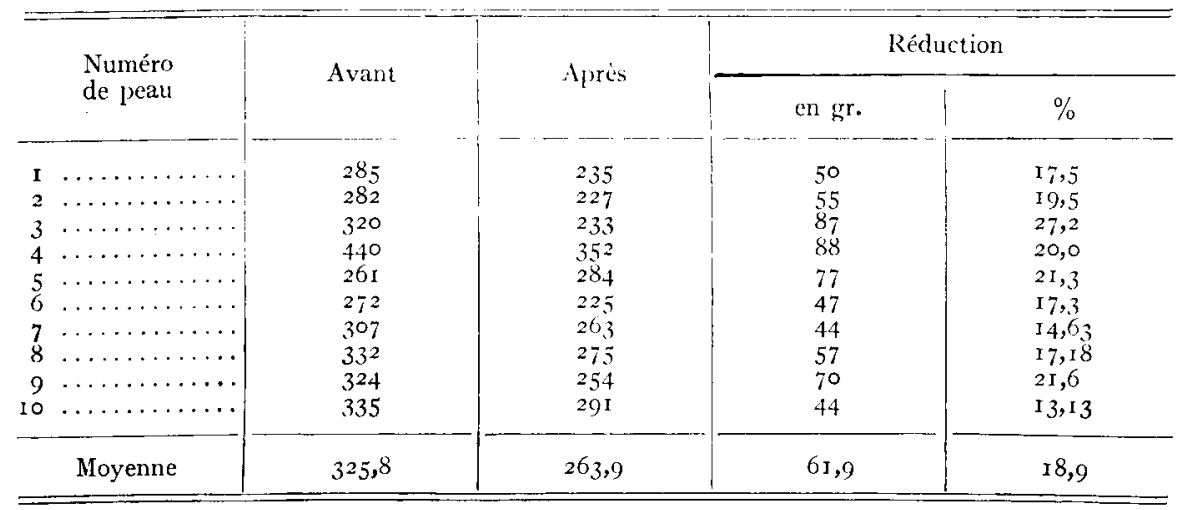


Le maximum de variation de poids est enregistré pour les peaux afghanes et russes qui subissent un écharnage incomplet au moment $\mathrm{du}$ sacrifice des agneaux. D'autre part le confit prend une part importante dans leurs poids.

Les peaux sud-africaines, par contre, sont beaucoup plus légères car elles sont simplement séchées sans confit. De plus le premier écharnage est effectué avec soin de sorte que la variation de poids au cours du tannage n'est en moyenne que de 4,8 p. roo alors que pour les peaux confitées elle atteint I8,9 et 27,4 p. IOo (peaux russes et afghanes).

Il est à noter que le poids des peaux dépend de leur surface, de leur épaisseur, de la densité de leurs tissus, de la densité pileuse, du confit, de la technique et degré de séchage et enfin des produits tannants employés.

\section{2o Peaux produites au C.N.R.Z.}

I,étude des peaux produites au C. N. R. Z. nous a permis de suivre toutes les opérations que les peaux Karakul subissent depuis la production jusqu'à l'utilisation. Nous avons pu comparer à l'état frais ce que nous n'avions pas pu faire pour les peaux commerciales. D'autre part nous avons pu mettre en évidence l'influence du mode de séchage sur les caractéristiques de la peau et sur sa qualité. Pour cela nous avons accéléré le séchage par une exposition de plus ou moins longue durée aux rayons Infra-Rouge tout en gardant quelques peaux témoins pour la comparaison. D'autre part l'épaisseur de la peau a été mesurée sur la tête, sur le cou et sur le ventre en plus des niveaux dont nous avons parlé à propos des peaux commerciales obtenues sur le marché.

\section{a) Surface}

I a surface des peaux fraîches subit deux réductions : une première fois au cours du séchage et une seconde fois au cours du tannage. Pour déterminer l'influence du séchage sur la réduction de la surface, nous avons divisé les peaux en 4 lots : le premier servant de témoin, a été saupoudré de gros sel et séché simplement à l'air et à l'ombre.I,e seconda subi une exposition de courte durée ( 2 heures) aux lampes Infra-Rouges, suivie de séchage à l'air. Le troisième a subi une exposition relativement longue (4 heures), suivie de séchage à l'air. Le tableau XXVI montre les résultats obtenus pour ces trois lots. Un quatrième lot a été exposé aux I. R. beaucoup plus longtemps que les précédents (plus de 5 heures). Voici les résultats obtenus :

Lot témoin: La réduction de surface est faible (I2,7 p. IOo) au cours du séchage, mais assez forte au cours du tannage (I9,6 p. Ioo). La réduction 


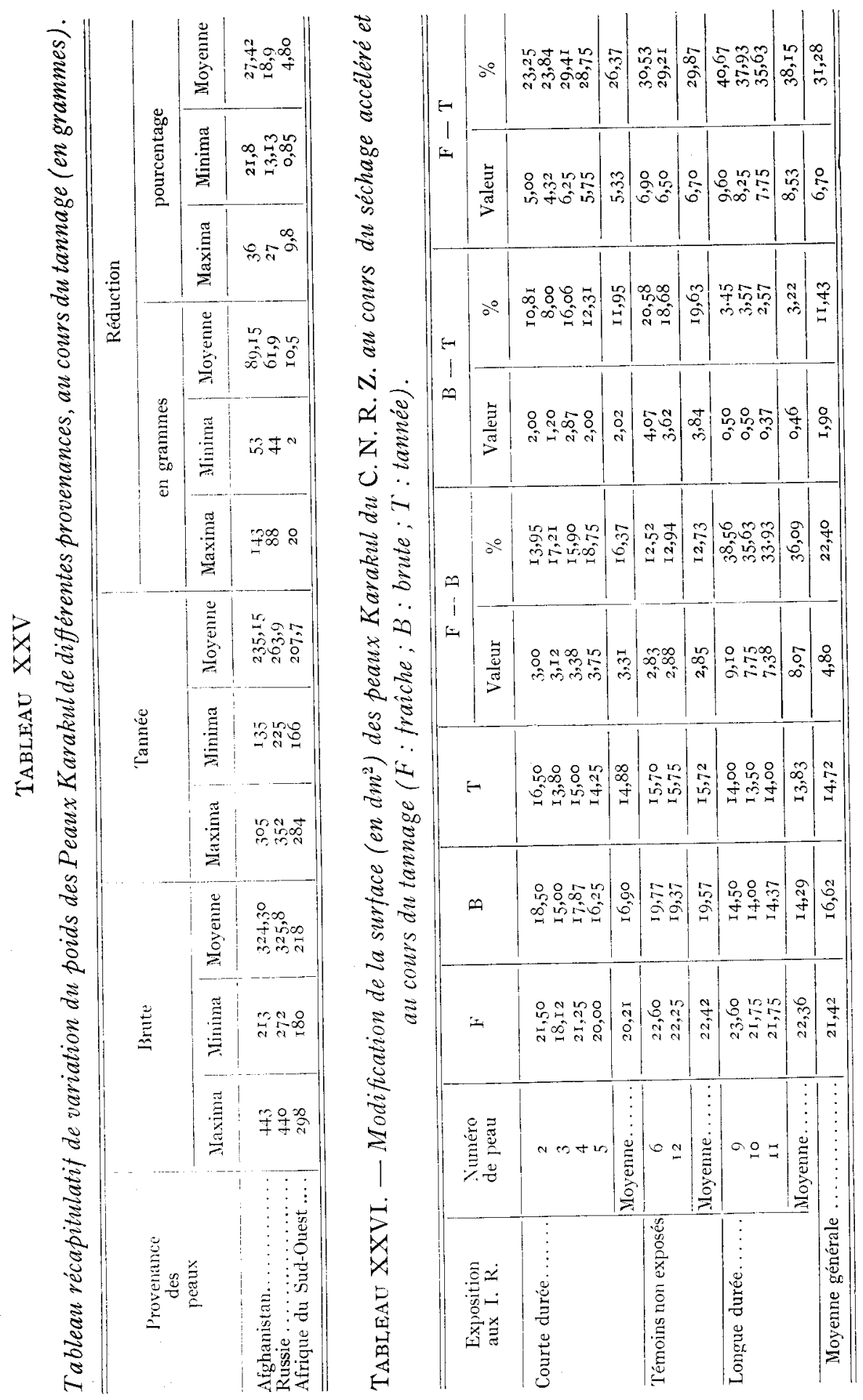


totale de la surface des peaux à la suite de ces deux opérations est de 29,8 p. IOO.

Lot $n^{0} 2$ : La réduction de surface est plus importante (I6,3 p. IOo) que dans le lot témoin, au cours du séchage mais beaucoup plus faible (Ix,9 p. IOo) au cours du tannage, de sorte que la réduction totale est légèrement plus faible $(26,3 \mathrm{p}$. Ioo) que celle du lot témoin.

Lot $n^{0} 3$ : La réduction au cours du séchage est très importante $(36$ p. roo) par rapport aux lots précédents, mais la réduction au cours du tannage est beaucoup plus faible $(3,2$ p. roo). Toutefois la réduction totale de la surface, à la suite des deux opérations, est plus importante ( $3^{8}$ p. roo) que dans les deux premiers lots.

Lot $n^{0} 4$ : I a réduction de la surface de la peau est très brusque et très importante, de 1'ordre de $44 \mathrm{p}$. Ioo, au cours du séchage ce qui altère la structure de la peau qui devient cassante et inutilisable. En effet les peaux constituant ce lot n'ont pas pu supporter le tannage et ont été déchirées en tous sens.

En comparant les résultats des 4 lots, on constate qu'un début de séchage accéléré (lot 2) n'affecte pas de façon très sensible la réduction totale de la surface des peaux. En effet les peaux ainsi traitées subissent une réduction totale peu différente du lot témoin.

Mais, si l'exposition aux I. R. est prolongée (lot 3 ), il se produit au cours du séchage une réduction plus importante de la surface, ce qui provoque une réduction totale également importante.

Enfin, si l'exposition aux I. R. est très poussée (lot 4), la superficie de la peau se réduit considérablement au cours du séchage. Ia peau ainsi traitée est fortement altérée et devient inutilisable.

\section{b) Epaisseur}

L'épaisseur de la peau fraîche est différente d'un point à l'autre de la surface du corps. Elle est maximum sur le dos $(\mathrm{r}, 62 \mathrm{~mm})$ et minimum sur le ventre $(0,87 \mathrm{~mm})$. Le tableau XXVIII montre l'épaisseur des peaux d'agneaux à différents niveaux du corps et pour les trois états de la peau (frais, brut et tanné).

Contrairement à la surface et au poids des peaux dont la variation au cours du séchage et du tannage est dans le même sens (réduction), l'épaisseur se réduit au moment du séchage puis augmente à nouveau durant le tannage. $L_{\text {a }}$ réduction est provoquée par le départ de l'eau de constitution de la peau. Celle-ci est graissée après tannage pour l'assouplir. Le graissage provoque en même temps le gonflement de la peau, ce qui explique l'augmentation de l'épaisseur. Les variations individuelles sont indiquées sur le tableau XXVII.

La variation de l'épaisseur n'est pas la même pour tous les points 

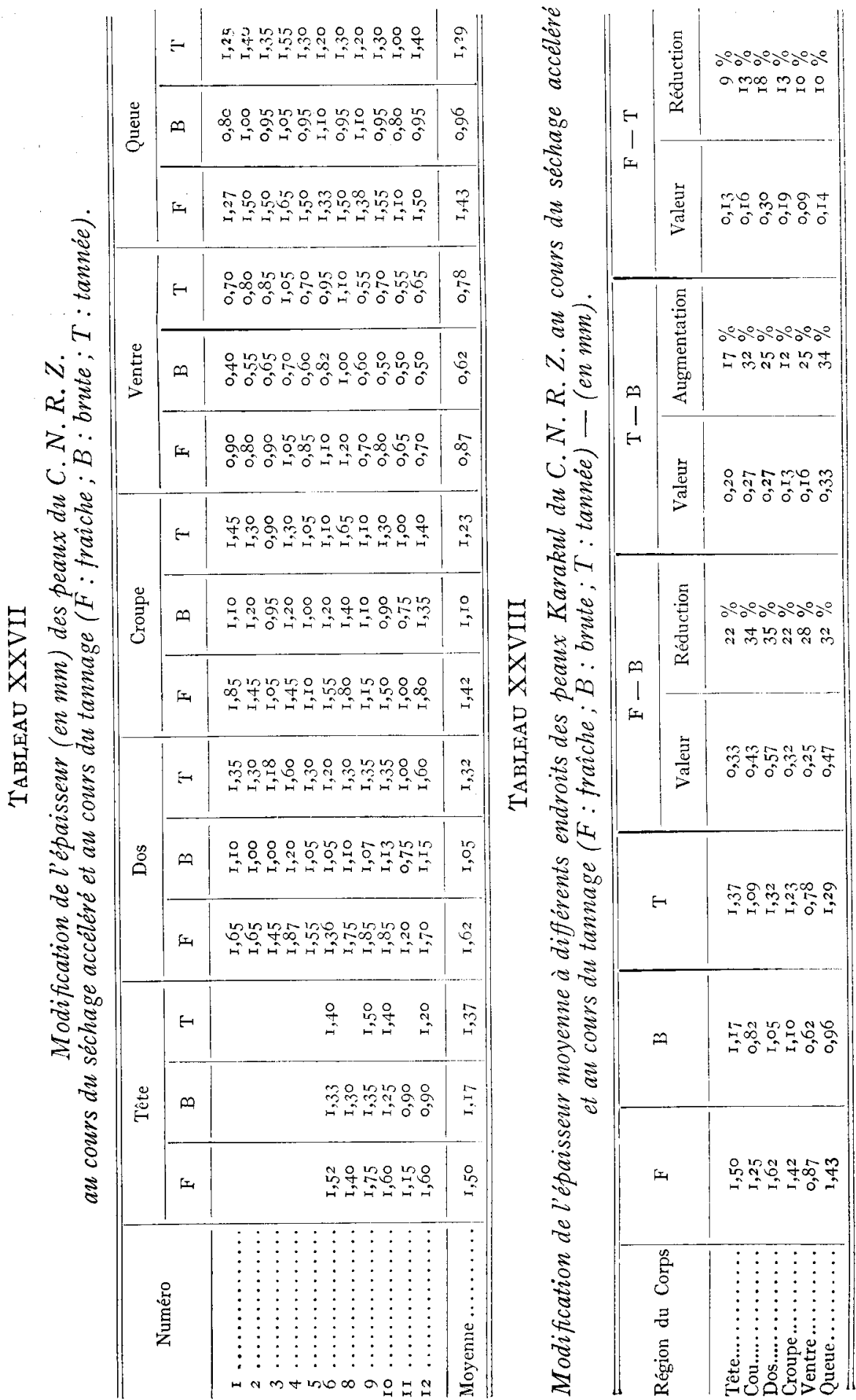
de la peau. C'est au niveau de la tête que la peau subit le moins de changement avec $22 \mathrm{p}$. roo de réduction due au séchage et I 7 p. Ioo d'augmentation au cours du tannage de sorte que la variation définitive se traduit par une réduction de $9 \mathrm{p}$. Ioo par rapport à l'épaisseur à l'état frais.

Par contre, le dos subit le maximum de changement avec $35 \mathrm{p}$. roo de réduction suivi de $25 \mathrm{p}$. Ioo d'augmentation. Le résultat final se traduit par une réduction de I8 p. Ioo par rapport à l'état frais.

Les variations, au cours du séchage et au cours du tannage, de l'épaisseur de la peau, ne sont pas proportionnelles à son épaisseur initiale. En effet l'examen du tableau XXVIII permet de constater que 1a réduction et l'augmentation de l'épaisseur au niveau du ventre où l'épaisseur initiale est minimum sont plus importantes qu'au niveau de la croupe par exemple. Par contre, la réduction définitive est plus importante au niveau de la croupe.

\section{c) Poids}

Chaque fois que la peau passe d'un état à 1'état suivant, son poids diminue. Ces réductions sont dues d'une part à la perte d'eau de constitution et d'autre part à l'écharnage et au grattage du côté interne de la peau. Les réductions partielles sont fortement influencées par le mode de séchage. L'examen du tableau XXIX montre qu'un séchage rapide (peaux $\mathbf{n}^{\circ} 9$ à II) provoque une réduction plus importante du poids $\left(5^{8,4}\right.$ à 64,5 p. IOo) alors qu'avec un séchage normal ( $n^{\circ} 6$ et $n^{\circ}$ I2) cette réduction ne dépasse pas 53,3 p. Ioo. Mais une forte réduction durant le séchage rapide est compensée par une réduction plus faible au cours du tannage. Ainsi les peaux $n^{\circ} g$ à $n^{0}$ II subissent, au cours du tannage, une faible perte de poids $(9,6-\mathbf{I} 6,9$ p. Ioo contre $22,6-33,2$ p. Ioo pour les peaux à séchage norma1).

\section{TABLEAU XXIX}

Modification du poids des peaux Karakul du C. N. R. Z. au cours du séchage accéléré et au cours du tannage ( $F$ : fraîche ; $B$ : brute ; $T$ : tannée) (en grammes).

\begin{tabular}{|c|c|c|c|c|c|c|c|c|c|}
\hline \multirow{2}{*}{$\begin{array}{l}\text { Numéro } \\
\text { de peau }\end{array}$} & \multirow{2}{*}{$\mathrm{F}$} & \multirow{2}{*}{ B } & \multirow{2}{*}{$\mathrm{T}$} & \multicolumn{2}{|c|}{ F-B } & \multicolumn{2}{|c|}{$\mathrm{B}-\mathrm{T}$} & \multicolumn{2}{|c|}{$F-T$} \\
\hline & & & & en $g$ & $\%$ & en $g$ & $\%$ & en $g$ & $\%$ \\
\hline $2 \ldots \ldots$ & $5^{82}$ & 282 & 186 & 300 & $5 I, 55$ & 96 & 34,04 & 396 & 68,40 \\
\hline $3 \ldots \ldots$ & 449 & 208 & I 44 & $24 \mathrm{I}$ & 53,67 & 64 & 30,77 & 305 & 67,92 \\
\hline $4 \ldots \ldots$ & 645 & 303 & 208 & 342 & 53,02 & 9.5 & $3 \mathrm{I}, 35$ & 437 & 67,75 \\
\hline $5 \ldots \ldots \ldots$ & 488 & 216 & I5O & 272 & 55,73 & 66 & 30,55 & 338 & 69,26 \\
\hline & $5^{68}$ & 264 & 186 & 303 & 53,34 & 78 & 29,67 & $3^{82}$ & 67,25 \\
\hline $9 \ldots \ldots$ & 465 & I 65 & 140 & 300 & 64,52 & 25 & I 5,15 & 325 & 69,89 \\
\hline го....... & $53^{\circ}$ & 207 & 187 & $3^{23}$ & 60,94 & 20 & 9,66 & 343 & $64,7 \mathrm{I}$ \\
\hline I $\ldots \ldots$. & 440 & 183 & $5^{2}$ & 257 & $5^{8,4 I}$ & $3 \mathrm{I}$ & 16,93 & 288 & 65,45 \\
\hline I $2 \ldots \ldots$ & 600 & 313 & 209 & 287 & 47,83 & 104 & 33,22 & $39 \mathrm{r}$ & 65,16 \\
\hline Moyenne & 529,66 & 237,88 & I 73,55 & $29 \mathrm{I}, 78$ & 55,08 & 64,33 & 27,04 & 356, II & 67,23 \\
\hline
\end{tabular}


Ainsi la perte totale de poids, c'est-à-dire la différence de poids entre l'état frais et l'état tanné définitif paraît indépendante du mode de séchage. Elle varie de 64,7 à 69,8 p. Ioo avec une moyenne de 67,2 p. Ioo.

\section{$3^{0}$ Modifications de la qualité de la iourrure.}

Les peaux tannées montrent peu de modification par rapport à leur état frais, mais le nettoyage permet aux boucles de se dessiner plus nettement.

Après le séchage (état brut), les peaux perdent apparemment une partie de leur qualité mais au cours du tannage l'aspect d'ensemble de la fourrure s'améliore comme le montre les photographies prises avant et après tannage: le dessin des boucles acquiert une netteté plus grande

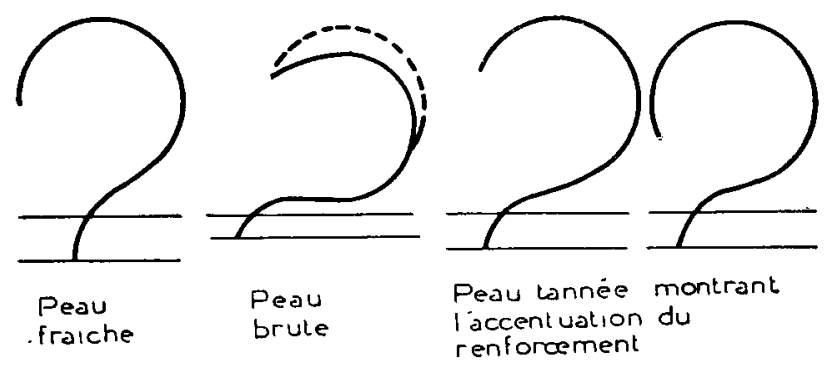

Fig. 65. - Schéma montrant le comportement de lit boucle suivant les variations de l'épaisseur de la peau aux stades Irais, brut et tanné.

et on a l'impression que les poils s'enroulent davantage. Le degré du lustre augmente également $\mathrm{du}$ fait du nettoyage et du frottement après graissage.

I'importance de cette amélioration de l'aspect des boucles varie avec les peaux et dans la même peau selon la région.

En ce qui concerne la raison de l'amélioration des boucles au cours du tannage, la formation des plis et rides sur la peau et le nettoyage des poils sont des facteurs essentiels. D'autre part, l'inclinaison des racines des poils aurait une influence certaine sur la fermeture des boucles. A l'état brut la peau se contracte au cours de la déshydratation, son épaisseur diminue et ce phénomène se répercute sur les racines des poils dont l'inclinaison augmente, ce qui éloigne le bout du poil de la surface de la peau (fig. 65).

Au cours de l'apprêt l'épaisseur de la peau augmente à nouveau, la racine du poil se redresse et le bout de la fibre se rapproche de la surface de la peau. Le départ des impuretés facilitant l'arrangement des poils, la boucle prend un aspect plus net et plus agréable. 


\section{DISCUSSION}

Il est assez difficile de comparer les résultats obtenus par les auteurs sur un matériel divers et subissant des traitements différents. En effet en dehors de la variété des familles à l'intérieur de la race Karakul, les méthodes de dépeçage, de séchage, de confitage et de tannage sont différentes d'un pays à l'autre. Il en résulte souvent des opinions divergentes et quelquefois mème incompréhensibles sans une longue interprétation. Ainsi FiröLICH et HorNísSCHEK (I94I) estiment entre I4 et 20 p. IOo la réduction de la superficie des peaux tannées par rapport à l'état frais. Nous-mêmes nous avons trouvé des réductions variant entre 23,35 et 30 , $50 \mathrm{p}$. IOo, pour les peaux ayant subi un séchage normal ou légèrement accéléré au début. Mais, la détermination de la surface des peaux fraîches constitue l'opération la plus délicate. Si on la calcule sur l'agneau vivant, elle ne peut être valable puisque certaines parties de la peau sont éliminées au moment de l'écharnage. Si on la détermine après dépeçage, elle varie suivant la manière dont elle est tendue. En effet, si on la tend à l'endroit, le côté intérieur de la peau colle à la table et garde la tension qu'on lui impose. Etendue à l'envers, elle a tendance à s'enrouler sur les bords.

La comparaison entre les peaux de provenance diverse montre, au cours du tannage, une augmentation de surface pour les peaux afghanes et russes ( 9,6 et 8 p. IOo). Par contre les peaux sud-africaines subissent une réduction de surface de 13,5 p. Ioo au cours de cette opération. Or la surface des peaux est un facteur essentiel de leur prix. Par conséquent on ne doit pas se baser sur la surface des peaux à l'état brut, car elle est susceptible d'augmenter ou de diminuer au cours du tannage.

L'épaisseur réduite des peaux est un autre facteur de la qualité. Or les peaux afghanes, épaisses à l'état brut, s'amincissent au cours du tannage alors que les peaux sud-africaines minces à l'état brut, s'épaississent au cours de cette opération de sorte que l'épaisseur à l'état tanné est sensiblement la même pour les deux.

En ce qui concerne le poids des peaux brutes, FröLICH, Herre et HORNITSCHEK (I938) avancent les chiffres de I4I-349 g pour la race pure et $185-377 \mathrm{~g}$ pour les agneaux croisés et une réduction de poids variant de 4 à 2 I p. Ioo par rapport à l'état brut. Nous avons trouvé :

2I3-443 g (moyenne 324,3 g) pour les peaux afghanes;

272-440 $\mathrm{g}$ (moyenne $325,8 \mathrm{~g}$ ) pour les peaux russes ;

I $80-298 \mathrm{~g}$ (moyenne $2 \mathrm{I} 8 \mathrm{~g}$ ) pour les peaux S. W. A.

Les réductions de poids après tannage sont respectivement 27,4 , I 8,9 et 4,8 p. Ioo par rapport à l'état brut. Il en résulte dans les peaux brutes afghanes un poids superflu dont le prix de transport est relativement important et que l'on pourrait éviter en abandonnant le confitage. 
On pourrait objecter que la mise en confit qui est en grande partie responsable du poids supplémentaire, assure une bonne conservation des peaux durant le transport et que, d'autre part, la mise en confit doit se faire de toute façon avant le tannage. Mais il ne faut pas oublier que le tannage seul ou avec la mise en confit se fait au même tarif par les firmes industrielles. De plus les peaux non confitées sud-africaines subissent parfaitement le transport sans aucune altération. Il s'agit simplement de bien assurer le séchage et la préservation des peaux contre les insectes dans les entrepôts et pendant le transport.

Quant aux modifications de la qualité des peaux, nos résultats confirment ceux de Judis (r933) en ce sens qu'il y a peu de changement d'aspect de la fourrure entre l'état frais et l'état tanné. Mais nous n'avons pas constaté de changement d'orientation des boucles, comme 1'a affirmé Kononova (I937). Nous n'avons non plus observé sur le matériel qui était à notre disposition, la détérioration des boucles orientées vers la queue, comme l'ont rapporté Frölich et HorNíscheck (I94I) et 'THOMpson (1938).

Par conséquent nous ne pouvons pas affirmer la détérioration, au cours du tannage, des boucles orientées vers la queue. Mais tout ce que l'on peut dire à propos de ces boucles c'est qu'elles sont indésirables. Car en général la majorité des boucles étant orientées vers la tête, les fourreurs ont pris 1'habitude de brosser les peaux de la queue vers la tête et de disposer, dans un manteau astrakan, les têtes des peaux vers le bas du manteau. Il est donc logique de penser que si certaines boucles sont orientées vers la queue, autrement dit vers le haut du manteau, elles se détruiraient aussi bien par le brossage que par les intempéries.

\section{CONCLUSION}

Nous nous sommes attaché, au cours de cette étude, à déterminer l'influence des préparations que subit la peau avant d'être utilisée en pelleterie, sur des peaux de provenances très diverses, les méthodes de récolte et de conservation différant sensiblement d'un pays à l'autre. C'est là un aspect important qui a échappé à la plupart des auteurs.

\section{I. - Peaux commerciales.}

I,es peaux sud-africaines ne perdent au tannage que 4,8 p. Ioo de leur poids tandis que la perte pour les peaux russes et afghanes est de I8 à $27 \mathrm{p}$. IOo. Les conséquences économiques de cette constatation semblent importantes quand on s'imagine le poids superflu $(27,42$ p. Ioo du poids global des peaux) dont les Afghans s'imposent de payer le transport sur dix à vingt mille kilomètres qui séparent la zone de production de celle de consommation.

I'épaisseur des peaux brutes est plus faible chez les peaux sud-afri- 
caines que chez les peaux russes et afghanes qui sont confites. Mais au cours du tannage les peaux sud-africaines s'épaississent alors que les peaux confites s'amincissent, pour arriver finalement à une épaisseur sensiblement identique.

Les peaux sud-africaines ont, à l'état brut, une surface plus grande que les peaux confites (russes et afghanes), du fait qu'elles ont été étirées au moment du séchage.

Or, l'étendue de la surface est une qualité appréciable de la fourrure. Mais il faut tenir compte du fait que les peaux sud-africaines diminuent de surface (13,5 p. IOo), au cours du tannage, alors que la surface des peaux confites augmente de $9,6 \mathrm{p}$. Ioo pour les peaux afghanes et de 8 p. Ioo pour les peaux russes.

2. - Peaux fraîches.

La surface des peaux fraîches se réduit d'environ $I / 3$ au cours des opérations de séchage et de tannage.

Elles perdent les $2 / 3$ de leur poids au cours des opérations de séchage et de tannage, indépendamment du mode de séchage.

Leur épaisseur diminue, au cours du séchage de $35 \mathrm{p}$. Ioo au niveau du dos, et augmente à nouveau (25 p. Ioo) pendant le tannage par suite de l'incorporation du tannin et de l'huile dans les tissus, sans toutefois retrouver l'épaisseur initiale.

3. - Qualité de la fournure.

I a qualité des boucles, le lustre et l'aspect d'ensemble de la fourrure, s'améliorent au cours du tannage.I'amélioration de la qualité des peaux tannées est peu importante par rapport à leur état frais, mais beaucoup plus importante par rapport à leur état brut. Le départ des impuretés, l'inclinaison des follicules, la formation des plis dans la peau et le frottement après graissage, interviennent dans l'amélioration de la qualité.

I,es boucles orientées vers la queue se détériorent bien plus au moment de l'utilisation qu'au cours du tannage.

\section{CONCLUSION GÉNÉRALE}

Nous avons mis au point les méthodes de mesure et d'observation relatives à l'examen par transparence des préparations de peau épaisse, à la longueur des poils, à la détermination du pourcentage de duvet, à la mesure du lustre.

Nous avons suivi le développement de la peau et du système pileux chez l'embryon, l'agneau et l'adulte Karakul. I'analyse des courbes de fréquence des diamètres et principalement des longueurs, a permis de suivre l'évolution des différentes populations de fibres et spécialement de préciser les rapports existant entre les fibres primaires et secondaires, à la naissance et pendant le débouclage. 
Nous avons procédé à une étude comparative dans plusieurs autres races afin de dégager ce qui est original dans le développement de la toison du Karakul, ce qui nous a permis une explication d'ensemble de la formation de la fourrure bouclée de l'agneau Karakul à la naissance. Dans ses grandes lignes, le développement folliculaire suit les mêmes lois que celui des autres ovins : développement des follicules primaires seuls pendant la phase pré-triade puis des deux follicules latéraux de part et d'autre des précédents, aboutissant à la formation des triades, enfin apparition des follicules secondaires dans l'angle de la triade.

Les caractères distinctifs essentiels de la toison du Karakul sont les suivants :

$\mathrm{I}^{\mathrm{O}}$ la rapidité de la protophase : 1e développement folliculaire chez le fœetus est très rapide durant la phase pré-triade, mais se ralentit déjà à la phase triade.

$2^{\circ}$ la lenteur de la néophase, se traduisant par:

a) un rapport $\mathrm{S} / \mathrm{P}$ très faible,

b) des fibres secondaires (laine) de longueur nettement inférieure à la moitié de celle des fibres primaires.

$3^{\circ}$ la persistance d'un caractère primitif de la toison à la naissance. Le follicule primaire central ne produit que des poils hétérotypiques ou des poils, et les follicules latéraux que des poils. Seuls les follicules secondaires produisent de la laine; nous avons retrouvé dans ces fibres la structure hélicylindrique qui a été décrite par d'autres auteurs; cette structure entraine la frisure de la fibre.

$4^{\circ}$ l'apparition dans la peau de secteurs au sein desquels les follicules restent parallèles entre eux, mais présentent des orientations différentes de celles des follicules des secteurs voisins. Ces secteurs apparaissent progressivement pendant le cinquième mois de la vie embryonnaire et tendent à s'effacer à la fin de cette période.

$5^{\circ}$ l'incurvation $d u$ poil pendant la fin de la vie embryonnaire qui coincide avec celle du follicule, sans qu'il soit possible de préciser celui des deux qui entraîne la courbure de l'autre. A la naissance, le redressement de l'ensemble est simultané.

Ce sont ces caractères originaux qui permettent d'expliquer 1'aspect particulier et l'état transitoire du pelage de l'agneau Karakul, car la formation de la boucle n'est pas due uniquement, comme l'ont pensé la plupart des auteurs, à la simple incurvation des poils, mais également:

- à la participation exclusive des poils et des poils hétérotypiques non frisés à la formation de la boucle, les brins de laine peu nombreux étant très courts à la naissance ;

- à la disposition des follicules qui permettent aux poils de croitre en s'accompagnant les uns les autres, dans des ensembles réguliers qui forment les boucles ; 
- à certaines conditions de l'environnement pendant les derniers temps de la vie embryonnaire, température, liquide amniotique, pression des enveloppes, etc. qui peuvent avoir une certaine action en accentuant la courbure du poil lors de sa croissance.

Après la naissance l'effacement du bouclage tient :

- à la poussée rectiligne des fibres primaires (poils), dont la partie incurvée constituant la boucle initiale, s'éloigne de la surface de la peau;

- à la croissance des fibres secondaires frisées qui participent de plus en plus à la constitution de la toison ;

- à la disparition des secteurs d'orientations des follicules, ceux-ci retrouvant leur parallélisme général du début de la vie embryonnaire ;

- à la suppression des conditions de l'environnement fœtal.

Ainsi, nous avons apporté une explication d'ensemble à la formation de la boucle, mais nos recherches ont également porté sur le problème de la qualité de la fourrure, cet aspect économique étant essentiel pour l'orientation de la sélection.

Chez l'agneau à la naissance, les corrélations que nous avons établies, montrent que les grosses boucles qui sont peu appréciées, possèdent les fibres les plus fines et les plus longues.

Or, il existe également une corrélation positive et significative entre la longueur des poils et l'épaisseur de la peau. Si ces caractères persistent chez 1'adulte - ce qui reste à vérifier - la sélection pour la qualité de la fourrure peut donc se faire à partir d'animaux à peau mince et à poil de gros diamètre. L'appréciation de ces 2 valeurs est aisée par des méthodes objectives; elle est donc utilisable dans la pratique de l'élevage du Mouton Karakul.

Nous avons également cherché si les opérations industrielles que subissent les peaux ne modifiaient pas les caractères appréciés à la naissance par l'éleveur.

I a qualité de la fourrure diminue après le séchage et le confitage, mais le tannage l'améliore.

Selon le traitement utilisé pour permettre le transport des peaux, des variations sont enregistrées après le tannage : c'est ainsi que les peaux brutes, séchées, mais non confites (peaux sud-africaines) diminuent de surface et augmentent d'épaisseur, tandis que les peaux confites (peaux russes et afghanes) ont une plus grande surface après le tannage. Mais ces variations compensent souvent les variations inverses qui se sont produites pendant le séchage ou le confitage. On peut donc dire que le confitage n'offre pas en définitive d'avantages appréciables, il entraîne en revanche un poids mort unitaire plus élevé qui grève inutilement les frais de transport.

Enfin nous avons tenté de déterminer par mesure optique la valeur du lustre, malheureusement les appareils utilisables ne peuvent donner 
qu'une appréciation d'ensemble, alors que l'œil apprécie des nuances qui nous amènent à une estimation de la qualité et de la beauté de la fourrure de l'agneau Karakul, qui résultent d'un ensemble unique et transitoire de conditions que l'on ne rencontre pas dans les autres races ovines.

\section{ANNEXES}

Les peaux d'agneau Karakul se présentent sous des aspects très divers dépendant de l'état de développement et du dessin de la boucle, de la couleur, du lustre, etc..., si bien que la liste définissant les différentes qualités est assez longue. Cette nomenclature est d'autant plus compliquée que la même peau est désignée sous plusieurs vocables, ou bien un même terme peut désigner plusieurs qualités différentes.

C'est pourquoi il nous a semblé utile de joindre cette annexe où seront définis les différents termes utilisés dans la production et le commerce des peaux provenant de l'agneau Karakul.

\section{I. - TERMINOLOGIE ZOOTECHNIQUE ET COMMERCIALE Io Karakul :}

C'est un terme turkmène ayant plusieurs origines possibles:

- Rose noire : les boucles de la fourrure sont appelées roses par les indigènes (Kara $=$ noir, $\mathrm{Kul} \mathrm{ou} \mathrm{gul}=$ rose ou fleur).

- Lac noir : les ondulations brillantes de la fourrure représenteraient des ondes brillantes d'un lac de couleur sombre.

- Nom d'une ville de la République de 'Turkmenistan.

Ce terme est couramment utilisé par les zootechniciens pour désigner l'" Ovis aries platyurae var. Karakul ", comme nous l'avons vu précédemment.

En France, on emploie fréquemment le terme de Boukhara pour désigner cette race. Nous lui préférons le mot Karakul qui a un caractère international, et évite la confusion avec d'autres races ovines de la région de Boukhara.

Dans le commerce de la fourrure on entend par "peau Karakul", la peau de l'agneau de cette race, celle de l'adulte n'étant pas utilisée en pelleterie.

\section{$2^{\circ}$ Astrakan :}

I,es avis sont partagés quant à l'origine de ce terme. Certains prétendent qu'à l'Exposition Internationale de Paris, en Igoo, qui suivit de près la visite du Tsar, un fourreur exposa des objets confectionnés en 
peau Karakul ; mais comme la garde du Tsar, une unité originaire de la ville d'Astrakan, était coiffée de toques en fourrure bouclée, il dénomma Astrakan ses objets en peau Karakul. Et cette dénomination a été adoptée par tous jusqu'à ce jour (M. MARGUELIES). D'autres rapportent qu'avant I88I, début de la conquête du Turkestan par les Russes, les peaux récoltées venaient de Boukhara par caravanes jusqu'à Astrakan. Eilles étaient embarquées à ce port de la Volga et remontaient ce fleuve par bateaux jusqu'à la foire de Nijny. Mais dès l'origine de ce commerce, les Russes avaient adopté pour les peaux le nom indigène " karakoul ». Ce nom, à ce moment, n'évoquait rien pour les Français, pas plus d'ailleurs que le lointain " khanat " de Boukhara ; aussi ont-ils retenu comme dénomination de la marchandise, le nom du port d'embarquement qui leur était plus immédiatement connu.

L'Astrakan est donc un terme russe qui, comme le Karakul a plusieurs origines :

- Nom d'une province de la Russie d'Europe et celui de sa capitale à $90 \mathrm{~km}$ de la Mer Caspienne.

- Nom d'une race ovine à fesses grasses de Kirghiz, appelée Mouton Astrakan dont la fourrure d'agneau est appelée " Dreibel » ou " Treibel " (d'AigniaAux, I928).

En pelleterie, 1'Astrakan est défini de la façon suivante par la Chanbre syndicale des Fourreurs et Pelletiers français :

"L'Astrakan c'est la peau d'agneau d'origine asiatique sacrifié généralement à 1'âge de 2 à Io jours, à laine résistante, moirée, ou le plus souvent bouclée naturellement, produit direct d'" Ovis platyura " (Boukhara, Afghanistan) ou appartenant à cette espèce soit par ses caractères ancestraux (Mésopotamie, Shiraz), soit par sélection et croisements plus ou moins continus d'Ovis platyura avec des espèces locales (Roumanie, Pologne, Crimée, sud de la Russie, Caucase, élevages divers en tous pays, notamment l'Afrique du Sud). ")

I'Astrakan moiré, appelé Breitschwanz en allemand, Broadtail en anglais, Téker en afghan et Karakoultcha en turkmène est la peau d'agneau d'Ovis platyura ou de sa descendance, comme il est dit pour l'astrakan, né avant terme par cause naturelle (circonstances atmosphériques défavorables, maladie de la mère ou de l'embryon, avortement, etc.) et qui présente trois stades :

I. - Galyac $=$ Lessac $:$ stade peu développé de l'embryon (avant $4^{\mathrm{e}}$ mois) ;

2. - Breitschwanz $=$ Téker : deux ou trois semaines avant terme ;

3. - Astrakan-breitschwanz ou Persian-broadtail : quelques jours avant terme (fig. 66).

Nous tenons à préciser, à la suite de nos travaux, que le galyac ou lessac est la peau du fœtus Karakul âgé de II 5 à I25 jours dont la Annales de Zootechnie. - 1958. 
fourrure est lisse et luisante (fig. 67). I,e breitschwanz ou téker est la peau du fœetus Karakul âgé de I25 à I 45 jours et qui présente un pelage ondulé et un début de formations bouclées (fig. 68).

$\mathrm{Ne}$ devraient être considérées comme astrakan que les peaux d'agneaux présentant au moins un certain pourcentage de sang pur Karakul. Il serait préférable d'ajouter les termes pur sang ou croisé.

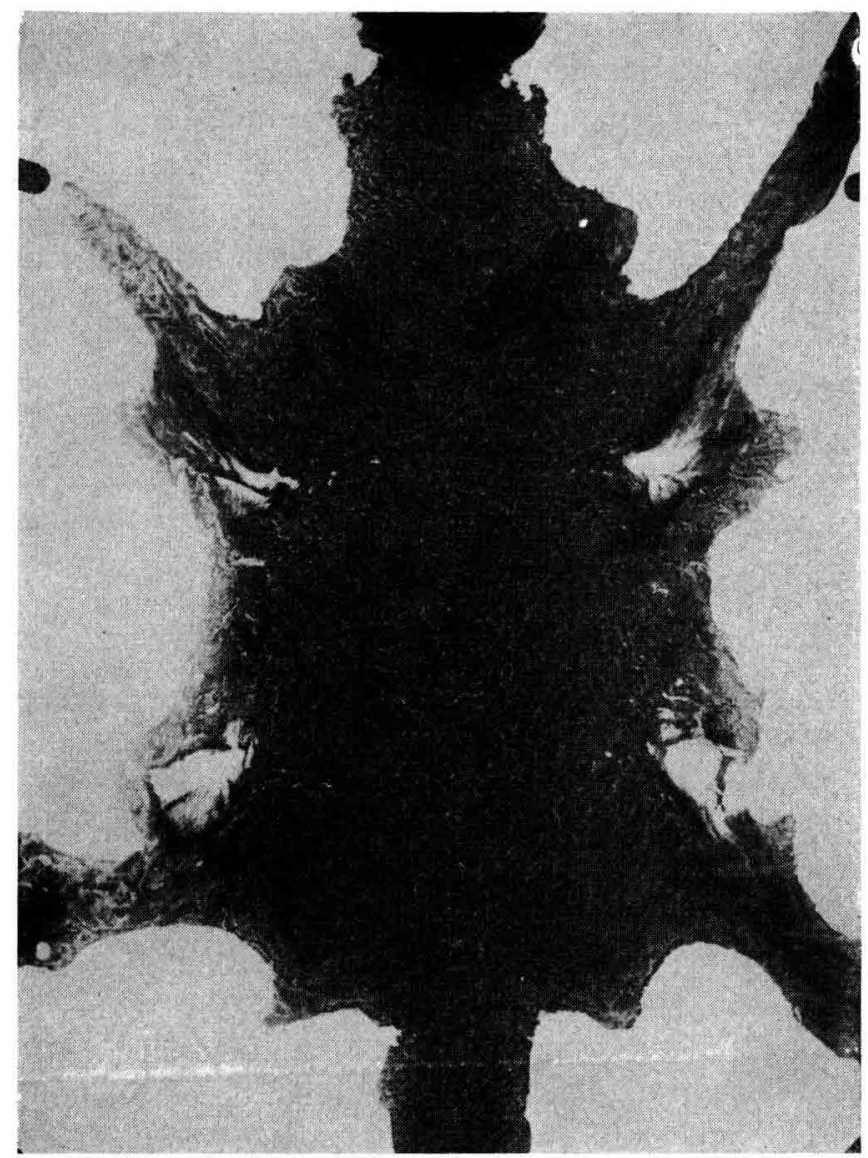

FIG. 66. - Peau Karakul afghane du type à boucles plates.

Mais nous reconnaissons que dans le commerce des peaux il est extrêmement difficile d'imposer ces additifs étant donné que la moitié des peaux commercialisées sont d'origine sud-africaines, done issues de croisements et que les producteurs de ce pays ne voudraient en aucun cas que leurs peaux soit affligées du mot "croisé. "

\section{$3^{\mathfrak{x}}$ Persianer}

Terme allemand de l'astrakan. 


\section{$4^{\circ}$ Persian lambskin}

Terme anglais de l'astrakan. I à encore on peut se demander pourquoi ces termes différents, et quel rapport il y a entre l'astrakan et la Perse. Plusieurs explications répondent à cette question. Certains affirment qu'il a été appelé ainsi parce que dans les temps anciens les peaux d'agneau de ce type étaient envoyées en Perse et rendues sur les marchés

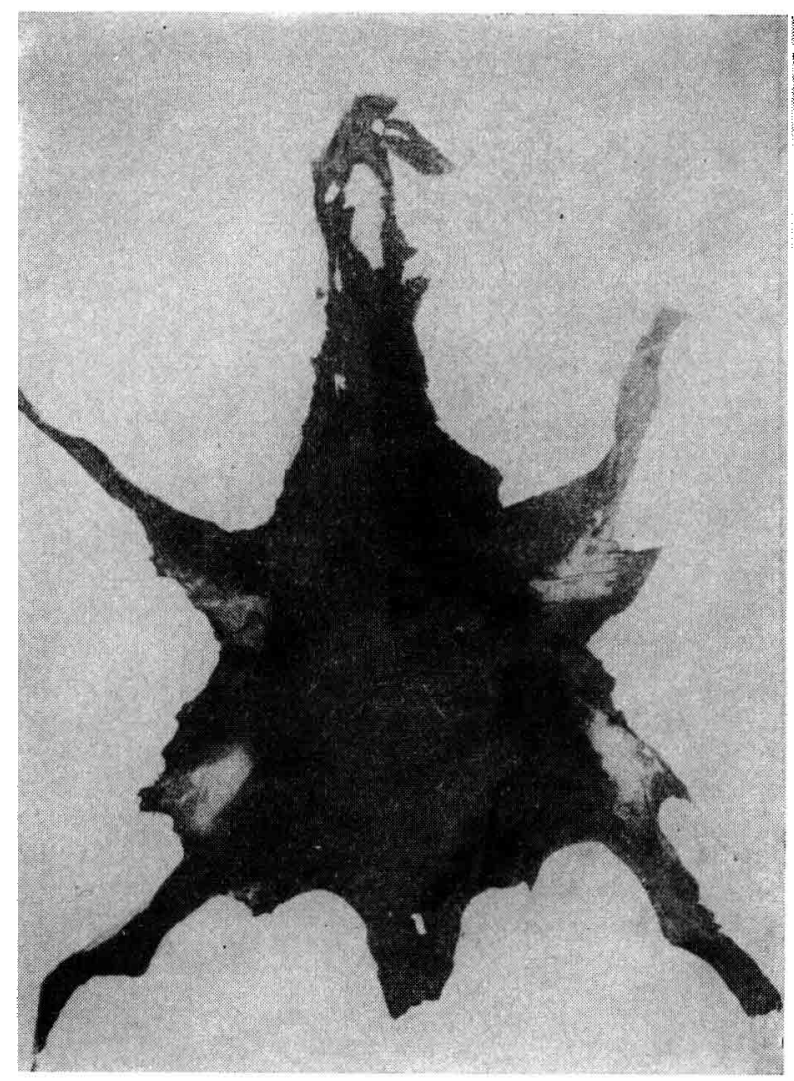

FIs, 67. - I'eau Kintalul afohane du lype Cialyac: I stac.

de ce pays. D'autres déclarent que cette fausse appellation est née parce que cette fourrure a été introduite en Russie d'Europe d'abord par les marchands persans, qui, en tant que mahométans, pouvaient se rendre à Boukhara, voyage qui était interdit aux chrétiens.

Une troisième explication nous apprend qu'il y a longtemps, alors que les communications entre l'Europe et l'Asie étaient à la fois difficiles et hasardeuses, des négociants a menaient des peaux d'agneaux bouclés aux foires de Russie d'Europe en particulier à Nijny-Novgorod et les appe- 
laient " Agneaux de Perse " afin de garder secrètes leurs sources d'approvisionnement.

Enfin, une quatrième explication peut être trouvée dans 1'histoire : avant l'ère chrétienne, l'empire perse, au summum de son influence culturelle et économique, et de sa puissance militaire, étendait sa domination sur une vaste région qui comprenait la plus grande partie de

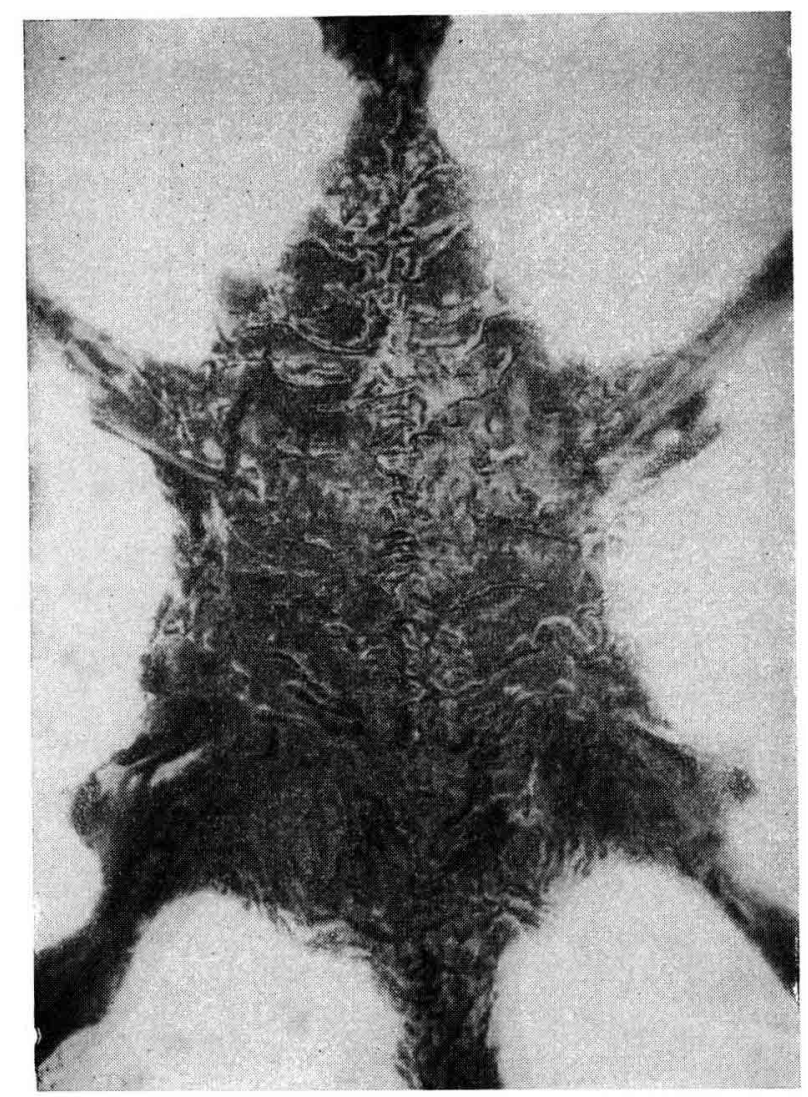

Fig. 68. - Peau Karakul afghane du tỵe Breitschwanz = Télier.

1'Asie Centrale. Toutes les productions de cette région, à cette époque et même plus tard, étaient appelées persanes ou de Perse, de sorte que les peaux bouclées d'agneaux Karakul, originaires de l'Asie Centrale, furent appelées à tort "peaux d'agneaux de Perse. "

Ces quatre versions peuvent être vraies à la fois, car elles se ramènent à la même idée : l'origine de ces peaux étant le bassin de 1'Amou-Darya, elles ont été appelées à tort "peaux d'agneaux de Perse. " 


\section{$5^{\circ}$ Demi-Persianer}

Terme vague, signifiant astrakan croisé ; peu importe le degré de dominance de la race pure : autrement dit, on peut appeler ainsi les croisements $I / 2,3 / 4,7 / 8$, etc.

\section{$6^{\circ}$ Shiraz}

Ce terme vient du nom d'une ville de l'Iran.

Dans le commerce des fourrures on appelle ainsi la fourrure d'agneau de couleur grise de la race Karakul ou d'autres races à boucles. Mais il est préférable d'appeler persianer gris les peaux d'agneaux Karakul gris, puisque dans les nomenclatures récentes de Karakul on ne trouve nulle part le terme Shiraz. On réservera donc cette dernière appellation pour des fourrures d'agneaux gris autres que Karakul; notons toutefois que certains auteurs anciens tel que ADAMETZ, on décrit le Karakul gris sous 1'appellation Shiraz.

\section{$7^{\circ} \mathrm{Kum}$}

Peaux à pelage bouclé de moutons à queue adipeuse originaires du sud de la Perse. Elles peuvent être noires ou grises, mais elles sont de qualité très inférieure à celle de l'astrakan.

\section{$8^{\circ}$ Peau de Salz (Salzfelle)}

Peaux de moutons à queue adipeuse, provenant d'Trak, de Syrie et de Palestine, et de qualité inférieure à celle de l'astrakan.

\section{II. - PRODUCTION ET COMMERCIALISATION DE LA FOURRURE D'AGNEAU}

\section{$\mathrm{I}^{0}$ Sacrifice des agneaux}

Nous avons vu que la toison de l'agneau Karakul commence à se déboucler dès la naissance. Toutefois, il y a des agneaux nés avec un état de boucle sous-développé et d'autres avec un état sur-développé. I1 y a donc, pour chaque agneau, une phase optimale correspondant au meilleur état de la boucle. Les facteurs principaux du débouclement étant le mouvement de retour des follicules et la croissance des poils, il faut donc saisir ce moment optima1, pour fixer la boucle. On sait que la phase où la boucle atteint son maximum de beauté, de brillance et de solidité n'est pas le seul facteur intéressant. Le commerce porte également un grand intérêt à la surface des peaux. Par conséquent on est contraint de tenir compte d'une part de la phase critique de la fermeté de la boucle 
où intervient également le facteur individuel, et d'autre part, de la taille des peaux, qui augmente avec l'âge. Ceci pose des problèmes quant à l'âge de sacrifice des agneaux. Ces difficultés sont faciles à surmonter dans les petits troupeaux issus de familles homogènes, les différences d'un individu à l'autre étant peu marquées.

Quant aux grands troupeaux, la détermination de 1'âge de sacrifice a été l'objet de controverses. En réalité les règles établies par chaque auteur ne sont valables que pour les troupeaux observés et ne peuvent être généralisées. C'est ainsi que le moment opportun d'après KoEPPEL (I9I9) et MEHNERT (I92I) pour le sacrifice des agneaux est entre le $4^{\mathrm{e}}$ et le $8^{\mathrm{e}}$ jour, alors que pour OWen WAHL (I920) ce serait entre le $3^{\mathrm{e}}$ et le $4^{\mathrm{e}}$ jour. LASSEN (I92I) estime que le sacrifice doit intervenir un peu avant la phase optimale, et en accord arec Thompson, il préfère le sacrifice dans les 24 heures qui suivent la naissance.

L'opinion la plus justifiée à cet égard est, à notre avis, celle de IiröLICH et HORNITSCHEK (I94I) qui se résume ainsi :

I. - Le sacrifice doit se faire immédiatement après la naissance;

2. - 'Toutefois, les agneaux à poils courts et à petites boucles seront sacrifiés 2 à 3 jours après la naissance ; ils acquièrent ainsi la taille et la brillance sans préjudice pour la beauté et la fermeté de la boucle ;

3. - Dans les grands troupeaux, où il est difficile, faute de temps, de tenir suffisamment compte de ces considérations, il est conseillé de sacrifier les agneaux dans les 24 heures qui suivent la naissance.

Notons qu'en Boukharie les éleveurs ont coutume de sacrifier les agneaux entre le $2^{\mathrm{e}}$ et $1 \mathrm{e} 6^{\mathrm{e}}$ jour, plus fréquemment le $3^{\mathrm{e}}$ jour.

\section{$2^{\circ}$ Préparation des peaux}

Pour éviter que la fourrure soit tachée de sang, l'agneau sera suspendu par ses pattes arrières. Le dépouillement sera réalisé avec grand soin et en se servant le moins possible dı conteau a fin d'ériter des blessures de la peau qui la déprécieraient.

Après écharnage minutieux et lavage de la peau, on procède au séchage qui se fait soit sur le sol, soit sur des cordes, soit sur des cadres en bois. Après égouttage au soleil le séchage est poursuivi à l'ombre car une exposition prolongée au soleil provoquerait le " dédoublement" de la peau, c'est-à-dire la séparation de la partie contenant les follicules de la couche sous-jacente. En Afghanistan et en Boukharie, pour accélérer le séchage on applique une couche de gros sel contre la face interne de la peau. I,e sel évite d'autre part la putréfaction de la peau.

$\mathrm{Vu}$ la distance qui sépare la zone de production de celle de l'utilisation, les peaux sont souvent stockées assez longtemps en attendant le tannage. Ceci pose des problèmes de conservation durant le stockage 
et le transport. On parvient à les résoudre par le procédé très ancien du confitage qui consiste à tremper les peaux pendant quelques jours dans un bain de farine d'orge et de sel. Les acides organiques libérés par cette fermentation, ainsi que la couche de sel, de son et de farine qui adhèrent à la peau, garantissent sa conservation tout en facilitant le tannage ultérieur. A la sortie des bains, les peaux égouttées, séchées, et empilées cuir contre cuir et poil contre poil, sont stockées ou emballées pour le transport. Des produits antiparasites de toute nature sont utilisés contre les dégâts éventuels causés par les insectes.

En Afrique du Sud-Ouest, les peaux sont lavées, trempées dans un bain insecticide et simplement séchées à l'ombre sur des cadres en bois sans apport de sel ni de confit.

\section{$3^{\circ}$ Commerce et utilisation}

Les marchés internationaux des peaux Karakul sont centralisés dans certaines grandes villes telles que Léningrad, New-York et Londres où les peaux brutes sont vendues aux enchères par quelques firmes spécialisées et à certaines dates déterminées de l'année.

Chaque pays producteur présente ses marchandises, en général à l'état brut et classées selon sa propre classification. Le commerçant grossiste connaissant ses besoins et la classe de peau qui y correspond, procède aux achats par lots de roo peaux et plus.

Une fois tannées, les peaux subissent une seconde classification et sont vendues aux pelletiers qui les utilisent pour la confection de manteaux, vestons, revers et chapeaux. Les peaux galyacs servent dans la haute couture pour la confection de robes entières et de souliers.

\section{III. - LA BOUCLE DE LA TOISON D'AGNEAU}

Chez l'agneau Karakul, comme nous l'avons vu, seules les fibres primaires prennent part à la formation de la boucle, alors que les fibres secondaires, à peine sorties de la peau, forment le duvet. Parmi les fibres primaires, certaines servent de support à d'autres et ces deux groupes de fibres se croisent ou superposent tout simplement. Chezl'agneau Karaku1, quoiqu'un peu irrégulièrement, les boucles sont dans l'ensemble orientées vers la tête. C'est pour cette raison que les fourreurs brossent la peau en passant la brosse de la queue vers la tête pour éviter d'ouvrir les boucles.

L'examen des peaux tannées nous a permis de distinguer les boucles simples des boucles composées.

I. - Boucle simple : chaque poil participant à cette boucle recouvre toute la largeur de celle-ci. C'est le cas notamment des boucles en tube et leurs dérivés. 
2. - Boucle composée : chaque poil ne recouvre qu'une petite fraction de la largeur de la boucle. Ceci est caractéristique des peaux du type "tchakmaki" (fig. 69 et 64).

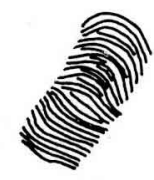

A

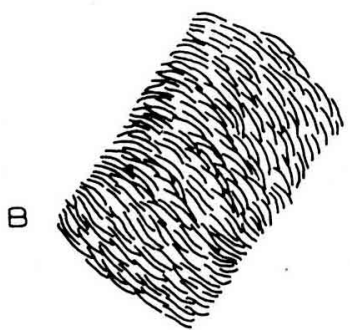

Fig. 69. -- Schémas montrant la différence entre une boucle simple (A) et une boucle composée $(B)$.

\section{A. - Caractéristiques de la boucle.}

\section{I ${ }^{\circ}$ Dimensions. Grosseur}

Dans la pratique on entend par grosseur la largeur de la boucle car cette caractéristique est la plus visible au coup d'œil de 1'observateur. Pour apprécier la largeur de la boucle on emploie les termes suivants ( $\mathrm{L}_{4} \mathrm{AN}-$ GLET et Collaborateurs, I947) :

Petite : Largeur inférieure à $4 \mathrm{~mm}$;

Mi-petite : Largeur égale à 4-5 $\mathrm{mm}$;

Moyenne : Largeur égale à 5-6 $\mathrm{mm}$;

Mi-grosse : largeur égale à 6-7 $\mathrm{mm}$;

Grosse : largeur supérieure à $7 \mathrm{~mm}$.

\section{$2^{\circ}$ Degré de fermeture}

Ce terme, introduit par TäNZER (I928) dans la littérature du Karakul signifie le degré d'enroulement du poil. Quand la courbure du poil est complète et forme un ? ercle, son degré de fermeture est égal à I (fig. 70). Les différents degrés utilisés sont $\mathrm{I} / 4, \mathrm{I} / 2,3 / 4$ et $\mathrm{I}$, qui correspondent à $I / 4, I / 2,3 / 4$, ou $4 ! 4$ de cercle. Plus le degré de fermeture s'approche de $r$, plus solide sera la boucle. Les dessins auront de ce fait un aspect net et la peau une valeur plus grande.

\section{$3^{\circ}$ Profondeur d'enroulement :}

Nicov (I929) a introduit ce terme pour exprimer le niveau auquel'commence la courbure du poil. La profondeur d'enroulement est fonction de la longueur du poil (fig. 7o). Plus la courbure est près de la surface de la peau, plus solide sera la boucle. La longueur du poil augmente avec 
l'âge et la partie enroulée du poil se sépare de la peau par un pédoncule. On apprécie la profondeur d'enroulement par des valeurs I, $3 / 4, \mathrm{r} / 2$ et
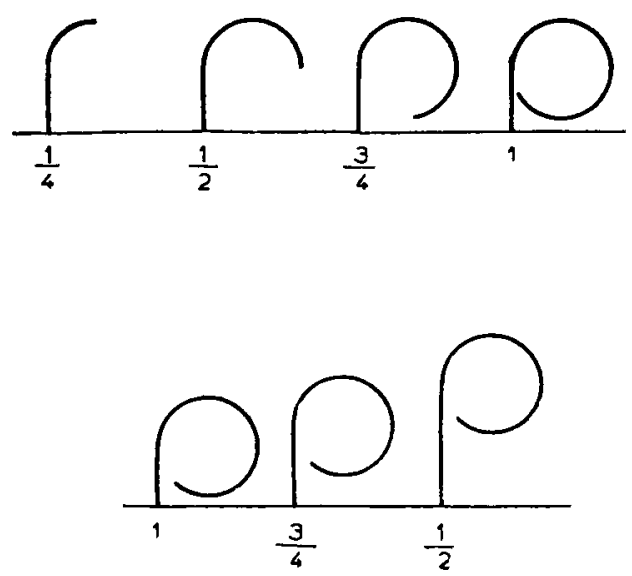

FIS , jo. - lin haut : degré de fermeture de la boucle.

En bas : profondeur d'enroulement de la boucle.

I/4. La valeur I signifie que le diamètre de la boucle est égal à la hauteur totale de celle-ci, alors que pour la valeur I/4, le diamètre est égal à $I / 4$ de la hauteur totale de la boucle.

\section{$4^{\circ}$ Axe de la boucle}

Le degré d'inclinaison de cet axe reflète en quelque sorte l'évolution phylogénique de la boucle. En effet, les boucles du type sauvage ont leurs axes perpendiculaires à la surface de la peau. Ainsi l'axe qui est perpendiculaire à la peau dans la boucle tire-bouchon, s'approche de la parallèle
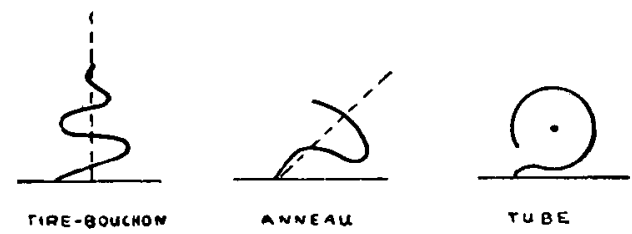

FIG. 7I. - En haut : axe de la boucle.

$E n$ bas : sens de torsion des poils constituant la boucle.

en passant par la série des types anneau, haricot, noix et tube (fig. $7 \mathrm{I}$ ). Autrement dit, le degré d'inclinaison de l'axe de la boucle pourrait être considéré comme un test de sélection dans le sens de l'obtention de la boucle en tube. 


\section{$5^{\circ}$ Sens de torsion}

Le degré d'enroulement (degré de fermeture) du poil, à lui seul, ne suffit pas pour expliquer la fermeture de la boucle. Fn effet, si la torsion est dans un certain sens la boucle sera fermée, alors que dans le sens opposé elle sera ouverte, (HILDPRANDT, I934), bien que l'inclinaison du follicule soit identique dans les deux cas (fig. 7 I).

\section{B. - Difiérents types de boucles.}

\section{Tube}

C'est la forme principale et la plus recherchée des boucles. Le tube est une boucle dont l'axe est parallèle à la surface de la peau et dont la
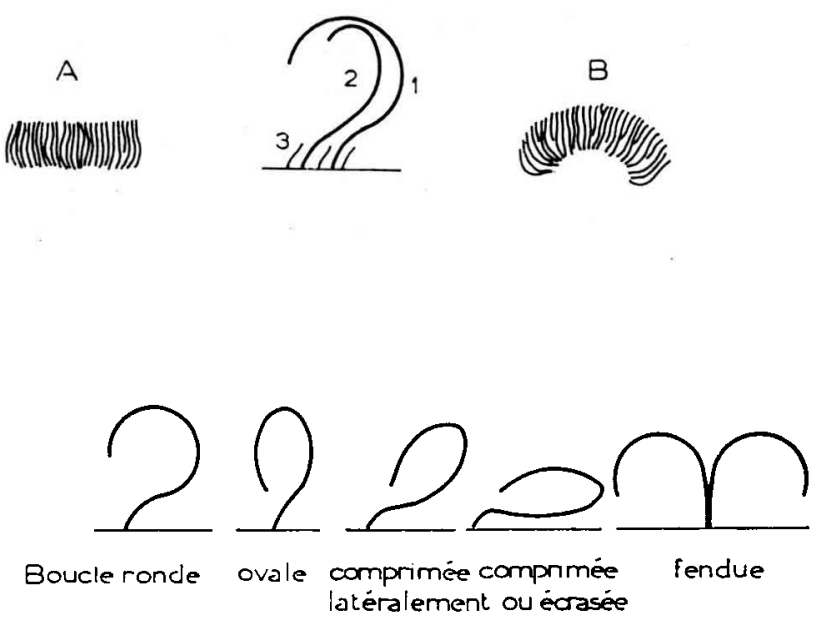

Fig. 72. - En hant : boucle en tube (A), boucle en haricot (B), poil de couverture (I) poil de soutien (2), duvet (3). En bas : état de la boucle.

forme cylindrique ressemble à un tube. Le tube est formé de deux groupes de poils. Les plus superficiels appelés poils de couverture ont une courbure circulaire plus ou moins parfaite et reposent sur le deuxième groupe appelé poils de soutien. Les deux groupes se croisent pour consolider la fermeté de la boucle (fig. 72).

Les tubes peuvent être plus ou moins longs et avoir des dispositions diverses.

a) Tubes parallèles: Ils sont parallèles entre eux sur une certaine portion de la surface de la peau.

b) Tubes mélangés : Ils sont disposés irrégulièrement les unes par rapport aux autres. 
c) Tubes rompus $=$ cassés: Ce sont des tubes très courts $\left(\mathrm{L}_{4}=0,5 \mathrm{~cm}\right)$ considérés comme résultant de la rupture de tubes plus longs.

d) Tubes horizontaux: Ils sont parallèles à la ligne dorsale de l'agneau.

e) Tubes verticaux: On appelle ainsi les tubes plus ou moins perpendiculaires à la ligne dorsale.

\section{$2^{0}$ Haricot}

C'est un tube dont 1'axe présente une courbure en forme de croissant. Sa 1ongueur est variable mais en général oscille entre I et I,5 cm (fig. 72).

Le tube et le haricot sont les deux types les plus intéressants de la boucle et très recherchés, aussi bien par le fourreur que par l'éleveur.

\section{$3^{\circ}$ Tire-bouchon}

Quand les poils d'une boucle s'enroulent à la façon d'une hélice ou a affaire à un tire-bouchon. En somme c'est une forme primitive et non sélectionnée. L'axe du tire-bouchon est plus ou moins perpendiculaire à la surface de la peau (fig. 7I).

\section{$4^{\circ}$ Faucille}

C'est une sorte de tire-bouchon dont le cercle distal est fermé à moitié, c'est-à-dire qu'il a un degré d'enroulement égal à $\mathrm{I} / 2$. C'est quelquefois le cas des agneaux nés avec un état sous-développé de la boucle.

\section{C. - Etat de la boucle.}

Les boucles que nous venons de décrire, notamment les tubes, peuvent être qualifiées de:

\section{$I^{0}$ Boucle ronde}

Quand la section transversale de la boucle a une forme circulaire (fig. 72).

\section{$2^{0}$ Boucle ovale}

Quand cette section a la forme ovale et que le grand axe de cette section ovale est perpendiculaire à la surface de la peau (fig. $7^{2}$ ).

\section{$3^{\circ}$ Boucle comprimée}

Quand la section a la forme ovale et le grand axe de cette section est parallèle à la surface de la peau (fig. 72). 
La boucle sera qualifiée de comprimée latéralement lorsque ce même grand axe formera un angle voisin de $45^{\circ}$ avec la surface de la peau.

\section{$4^{\circ}$ Boucle fendue}

C'est la figure formée par un ensemble de deux boucles en tube juxtaposées et séparées par un sillon très étroit. Les deux boucles ont des orientations inverses l'une de l'autre (fig. 72).

\section{$5^{\circ}$ Boucle plumeuse}

Si cette boucle fendue est écrasée, on l'appelle plumeuse du fait de sa ressemblance avec une plume d'oiseau.

\section{$6^{\circ}$ Boucle forte}

Une boucle est dite forte lorsqu'elle est ferme, élastique et dense. Le degré de fermeture est voisin de l'unité. Lorsqu'on passe la main sur la peau, les boucles présentent une certaine résistance et les poils déplacés par le mouvement de la main reprennent leur position initiale.

Si au contraire ils ne reprennent pas leur place et si la boucle a tendance à s'ouvrir, on a affaire à une boucle lâche.

\section{$7^{\circ}$ Boucle pédonculée}

Une boucle est dite pédonculée quand sa profondeur d'enroulement est inférieure à $3 / 4$, c'est-à-dire que le diamètre de la boucle est inférieur à $3 / 4$ de la hauteur totale. Les boucles surdéveloppées, notamment sur le cou de l'animal, sont pédonculées. L'extrémité du poil ne touche plus la surface de la peau; par conséquent une boucle pédonculée n'est pas ferme et forte. Le pédoncule peut être droit ou tortueux.

\section{$8^{\circ}$ Boucle jarreuse}

Dans une boucle jarreuse on voit des poils durs et grossiers qui dépassent la boucle du fait qu'ils n'ont pas la même courbure et élasticité. C'est un signe du manque d'homogénéité des poils, qui déprécie la qualité de la boucle.

\section{D. - Modèle de dessin de la fourrure.}

\section{Io Modèle en noix}

On désigne sous ce nom l'ensemble de plusieurs haricots et tubes courts, disposés de façon à donner l'impression d'une noix cassée en deux. Le terme noix a tendance à disparaître et être remplacé par ses composants haricots et tubes courts. 


\section{$2^{\circ}$ Modèle en lyre $=$ modèle étendu}

Il s'agit du dessin d'ensemble qui a la forme d'une lyre. Ce genre de dessin se trouve en général sur la croupe, la concavité de la lyre étant dirigée vers la tête. I,es boucles participant à la lyre peuvent avoir une section circulaire ou ovale.

\section{$3^{\circ}$ Modèle en bande $=$ en côte}

Des formations bouclées assez larges et très longues partent de la ligne dorsale et suivent les côtes de l'animal. Ce ne sont pas des boucles proprement dites car elles dépassent de beaucoup celles-ci en longueur et en largeur. Leur côté ouvert est dirigé vers la queue.

\section{$4^{\circ}$ Modèle fougère ou sapin}

I1 arrive parfois que les boucles plumeuses, étant fréquentes sur 1'agneau, se répartissent de la manière suivante : une série de boucles disposées bout à bout forment la ligne dorsale de part et d'autre de laquelle d'autres boucles plumeuses se détachent et suivent les côtes de l'animal. On a alors l'impression d'une feuille de fougère ou d'une branche de sapin.

\section{$5^{\circ}$ Modèle en vague}

On appelle vague le modèle présentant des dénivellations ressemblant aux vagues de la mer.

L'extrémité du poil en sortant de la peau retourne vers celle-ci puis la quitte de nouveau légèrement. Dans certains cas on peut considérer la vague comme un stade primitif de la boucle en tube. Dans ce cas, la vague évoluera au bout de quelques jours en tube sous-développé. Cependant ce n'est pas le cas général. I.es vagues se rencontrent souvent sur la tête, le ventre et les membres.

\section{$6^{\circ}$ Modèle moire $=$ Watered-silk}

Un modèle est dit moiré lorsqu'il présente les mêmes caractéristiques que le Breitschwanz, c'est-à-dire des zones lisses alternant avec des vagues. Le poil est court et l'ensemble a un reflet qui change avec l'incidence de la lumière.

L'agneau moiré est surtout connu depuis la naissance d'un agneau mâle à la Station expérimentale de Neudam en Afrique du Sud-Ouest, présentant tous les caractères du breitschwanz. A. D. Thompson l'appela Watered-silk. Ce modèle diffère du breitschwanz par ses formations bouclées un peu plus développées et par son origine. 


\section{$7^{\circ}$ Modèle à boucles plates $=$ shallow-curl}

C'est le stade un peu plus avancé du type moiré ; il forme la transition pour le passage au type boucle en tube.

Les vagues sont plus prononcées et les poils sont courts et ont la forme d'un S aplati et couché.

Le croisement moiré $x$ boucle en tube produit le type boucle plate (Thompson). I.es deux types moiré et boucle plate sont en général très lustrés.

\section{$8^{\circ}$ Modèle boucle}

Il s'agit d'un modèle ne présentant pas de boucles typiques mais qui donne toutefois l'impression de boucles.

\section{$9^{\circ}$ Modèle crépu $=$ tête de nègre}

I e modèle appelé crêpu ou tête de nègre comporte des formations bouclées, petites et feutrées conme les cheveux des nègres. I. es peaux de ce genre ne sont, en général, pas brillantes.

\section{Io $0^{\circ}$ Modèle flou}

Le terme flou est employé pour un modèle dont les boucles sont très mal délimitées et ne forment pas un dessin défini.

\section{II ${ }^{0}$ Modèle sauvage}

Un modèle est dit sauvage lorsqu'il n'offre aucun type de boucle ; cependant les poils ont une certaine incurvation. Il s'agit d'un mélange de formations bouclées grossières et de poils feutrés.

\section{I $2^{\circ}$ Champs défectueux}

On appelle champs défectueux des portions limitées ne portant pas de boucle, dans un modèle à boucles bien formées (fig. 54).

\section{I $3^{0}$ Miroir}

Quand le champ défectueux est complètement lisse, on a affaire à un miroir.

\section{I $4^{\circ}$ Modèle lisse}

Une portion de la peau est dite lisse quand elle ne porte que des poils couchés parallèlement à la surface de celle-ci, sans aucune incurvation 
ou avec une courbure très faible. En somme, c'est un miroir non entouré de zones à boucles. Souvent la face, les membres et le ventre de l'agneau sont lisses.

\section{I $5^{\circ}$ Modèle uniforme}

C'est un modèle présentant la même espèce de boucle.Le degré d'uniformité ne donne aucun renseignement sur la valeur et la qualité de la peau. Cependant une peau à boucles en tube et en haricot est davantage cotée si elle est uniforme.

\section{IV. - VARIÉTÉS DE LA RACE KARAKUL}

Le principal caractère distinctif des variétés de la race Karakul est le pigment contenu dans la toison. Il s'agit bien entendu de la toison d'agneau et non de celle de l'adulte.

Les différentes variétés se rencontrent dans les troupeaux Karakul avec les proportions suivantes (HILDPRANIT, I935) :

Variété noire : $75^{-8} 7$ p. IOO;

Variété grise : IO-I5 p. IOO;

Variétés colorées : 5-Io p. Ioo.

\section{$\mathrm{I}^{\mathrm{O}}$ Variété noire}

C'est la variété la plus répandue. Cependant il y a plusieurs nuances de couleur noire.

Esst-ce dû̀ à la quantité du même pigment noir, ou ces nuances sontelles en relation directe avec la constitution chimique du pigment ? On n'en sait rien. Tout ce que l'on peut dire à ce sujet, c'est qu'il y a des nuances depuis le noir bleuté, jusqu'au noir rouille, en passant par le noir intense, le noir grisâtre, le noir marron et le noir argenté à reflet métallique.

Le bleu-noir et le noir argenté sont en général beaucoup plus brillants que le noir grisâtre.

\section{$2^{\circ}$ Variété grise}

Dans la variété grise, la couleur est due à une juxtaposition de poils blancs et de poils noirs. I,eur proportion respective détermine la nuance du gris. C'est la fourrure de cette variété de Karakul qu'on appelle Persianer gris. Le terme Shiraz, souvent employé à tort, serait impropre car bien que chez le Shiraz également, il y a des poils blancs et des poils noirs, il s'agit d'une autre race ovine sans aucun rapport avec la race Karakul.

La boucle et le dessin du persianer gris ne sont, en général, pas de bonne qualité. Les poils sont longs, flasques, mous et peu brillants. D'autre part il y a un fort pourcentage de duvet. On a expliqué ce phénomène par 
des différences d'ordre morphologique et physiologique entre les poils noirs et les poils blancs. Ces derniers ayant un rythme de croissance plus rapide, dépassent en longueur les poils noirs et constituent ainsi une hétérogénéité qui nuirait à la qualité de la boucle. D'autre part ces poils blancs ont peu de tendance à se boucler.

Iwanov et ses collaborateurs (I932) ont étudié la longueur des poils chez les trois nuances du persianer gris (foncée, moyenne et claire). Ils ont remarqué que sur une courbe de fréquence des longueurs, la distribution n'est pas identique pour les poils blancs et les poils noirs. La courbe des premiers comporte deux sommets alors que pour les seconds il y a un seul sommet.

IWANOv admet que pour le persianer gris foncé, la longueur moyenne des poils blancs est moins grande que celle des poils noirs. Ceci n'est pas valable pour le persianer gris clair où la longueur moyenne des poils blancs est plus grande que celle des poils noirs. Par conséquent il y a deux facteurs qui déterminent la nuance claire ou foncée: d'une part les longueurs respectives des poils et d'autre part leur proportion.

Il est aisé d'admettre dans ces conditions, que tous les poils blancs n'ont pas un rythme de croissance plus rapide que les poils noirs.

HiLDirandT (I934) pense que si le persianer gris n'a pas les mêmes qualités de boucles que la variété noire, c'est parce qu'il n'a jamais été sélectionné car à Boukhara on abat toujours les agneaux gris mâles.

Cependant il est à noter que si cette sélection n'a pas été faite, c'est parce qu'il y a difficulté, voire quelquefois impossibilité à la pratiquer chez la variété grise.

Les boucles fréquemment rencontrées chez le persianer gris sont des types en anneau, en faux, en tire-bouchon, etc. La boucle en tube est rare, de sorte qu'à qualité égale, l'astrakan gris peut dans ce cas valoir beaucoup plus cher que le noir. En général les persianers gris de très bonne qualité, trouvent acheteurs sur place, et ne sont pas exportés.

\section{$3^{\circ}$ Variété Kambar}

Les agneaux Kambar naissent avec une toison marron plus ou moins foncée. La qualité de la boucle est du même ordre que celle de la variété noire. Tous les poils sont colorés de la même manière.

\section{$4^{\circ}$ Variété Sour}

Il s'agit encore d'une peau plus ou moins marron, mais les pointes des poils sont beaucoup plus claires que la partie moyenne, leur base étant plus foncée.

L'ensemble a $11 n$ reflet doré-argenté, avec une riche gamme de 
nuances très séduisantes. Ces peaux, achetées jusqu'à I5 fois plus cher que les noires sur le matché local, ne sont jamais exportées, ou le sont rarement.

Selon Iwanov (I932) elles pourraient valoir jusqu'à 20 fois plus cher que la meilleure qualité du persianer noir.

\section{$5^{\circ}$ Variété rose}

Les poils sont d'un rouge rouille, mélangés d'une certaine proportion de poils blancs. Il y a plusieurs nuances suivant l'intensité du pigment et la proportion des poils blancs. Ces derniers proviennent des follicules secondaires et ont un diamètre plus faible par rapport aux poils pigmentés.

\section{$6^{0}$ Variété abrache $=$ Gul-É-Gaz}

La toison des agneaux abrache est composée de poils blancs et de poils marrons ; l'ensemble présente un aspect gris brun. Sur les échantillons que nous avons examinés, tous les poils marrons semblent provenir de follicules primaires ; parmi les poils blancs nous avons rencontré aussi bien des primaires que des secondaires. Par conséquent leur nombre est de beaucoup supérieur à celui des poils marrons. C'est une variété assez rare et très prisée, donc assez chère.

\section{$7^{\circ}$ Variété blanche}

Les poils sont d'un blanc pur et uniforme. On peut rencontrer par-ci par-là quelques poils noirs, toujours de forte épaisseur. Il est très rare de trouver dans cette variété des boucles en tube ou en haricot. Les boucles les plus courantes sont du type anneau, en faux, et du type vague.

\section{$8^{\circ}$ Autres variétés}

En dehors de celles que nous avons énumérées jusqu'ici, on peut rencontrer d'autres variétés plus ou moins régulières, telles que chalili sellé (tache plus claire sur le dos) et d'autres avec les bordures plus claires ou avec des taches plus ou moins claires réparties irrégulièrement à la surface de la peau.

Il est à noter que partout où il $\mathrm{y}$ a juxtaposition de poils blancs et colorés, ces derniers sont très souvent des primaires et de forte épaisseur, alors que les poils blancs sont un mélange de primaires et d $\epsilon$ secondaires. 


\section{REFEERENCES BIBLIOGRAPHIQUES}

AdAmetz (L.), I9I4. - E1 Carnero Karakul. Ior pp. illus. Talleres de Publicaciones de la Direccion Meteorologica, Buenos Aires.

ADAMETZ (L.), I927. - Uber die Herkunft der Karakulschafe Bucharas und die Entstehung der Lockenbildung am Lammvliese dieser Rasse. Z. Tierzucht, 8, I-64.

Aliwörden (K. Z. ron), Igr6. - Die Eigenschaften der Schafwolle und eine neue Untersuchungsmethode $z u m$ Nachweis geschädigter Wolle auf chemischem Wege. $-Z$. angei', chem., 29, 77-78.

Astrbury (W. T.), I942. - X-rays and the stoicheiometry of the proteins with special reference to the structure of the keratin-myosin group. J. Chem. Soc., 337-347.

AUBER (L. P. D.), I950-5I. - The anatomy of follicles producing wool-fibres, with special reference to keratinisation. - Trans. Soc. Edinburgh, 62, part I, no 7, I9I-254.

BENZIE (D.), I95I. - X-Ray diagnosis of pregnancy in ewes. - Brit. Vet.J., $10 \%, 3-6$ et 106, 23I-234.

Burns (M.), I I949. - Study on follicle population in relation to fleece changes in lambs of the English Leicester and Romney breeds. J. agric. Sci., 39, 64-79.

Burns (M.), I953. - Observations on the follicle population of Blackface sheep. - J. agric. Sci., 43, 422-43I.

CARTLR (H. B.), I943. - Studies in the biology of the skin and fleece of sheep I3. - Bull. Coun. Sci. industr. Res. Aust., no 164, I-59.

Cuenca (C. I. de), I952. - Un microtome pour analyser la finesse des fibres de laine en coupe transversale. - Bull. I. T. F., 33, 59-62.

D'Aigneaux (G. P.), I928. - Méthodes d'élevage des animaux à fourrure, II, 267-402. Bureau Technique pour l'élevage des animaux à fourrure. Thonon (Hte Savoie).

D'Aigneaux (G. P.), I93I. - L'adaptation en France de l'Industrie du mouton de Boukhara. - Journée du mouton de Boukhara, 23-I72. Fid. Maison Rustique, Paris.

Diomidova (N. A.), 1954. - Développement embryonnaire de la peau et de la laine chez les moutons (en russe). - Bull. Acad. Sci. URSS (Sér. biol.) (nov. déc.), no 6, 62-7I.

DUCLERT (I.), I888. - Déterminisme de la frisure des productions pileuses. J. Anat. et Physiol., Io3-III.

DUERDEN (J. E.), I939. - The arrangement of fibre follicle in some mammals with special reference to the Ovidae. - Trans. $r$. soc. Edinburgh., 59, $763-77$ I.

FröLICH (G.), Herre (W.), HornirscheK (H.), I938. - Die Veränderungen des Karakul-Persianervliesses während des Zurichtens. - Kuhn Arch., 47, I-26.

Frölich (G.). - HoRnitscheK (H.), I94r. - Das Karakulschaf und seine Zucht. - F. C. Mayer Verlag München-Solln. - Traduction espagnole (E,1 Karakul) + appendice par Vicente Boceta Duran, 502 pp., Sindicato National de Ganaderia, I946. Madrid.

GABE (M.), I953. - Quelques applications de la coloration par la fuschine paraldehyde. - Bull. micr. appl., II, 3, I53-I62.

GaLPIN (N.), I935. - The prenatal development of the coat of the New Zealand Romney lamb. - J. agric. Sci., 25, 344-36o.

Grbss (H. F.), I938. - A study of the development of the skin and hair of the australian opossum Trichosurus vulpecula. - Proc. zool. Soc. London, $\mathrm{B}, 108,6 \mathrm{II}-648$.

HARDY (M. H.), I946. - The group arrangement of hair follicles in the mamma- 
lian skin. Notes on follicle group arrangement in I3 australian marsupials. - Proc. r. soc. Queensland, 58, I25-I48.

HARdy (M. H.), LyNe (A. G.), I956. - Proposed terminology for wool follicles in sheep. - Nature, 19\%, 705-706.

Hardy (J. I.), Simmons (V. L.), I943. - Ear measurements in relation to pelt thickness and fur characters of Karakul lambs. - J. animal Sci., 2, I46-I5I.

HeLMERSEN (von), I839. (Rappotté pat D'AigneAUX, I928).

HERRE, (W.), WigGER (H.), I939. - Die Lockenbildung der Säugetiere. Kuhn Arch., 52, 233-254.

HildPRANDT (R. von), I934. - - Ein Beitrag zur Kenntnis der grauen Varietät des Karakulpelzschafes. - Z. Tierzucht., 27, 447-450.

HildPRANDT (R.), I935. - Un essai de classification biologique des variétés de Karakul d'après la couleur de la toison des agneaux nouveau-nés. (En tchèque), (Résumé en allemand), Sborn. Csl. Acad. Zemëd, 10, I I3-I I8 (B).

HoFER (H.), I9I4. - Das Haar der Katze, seine Gruppenstellung und die Entwicklung der Beihaare. Arch. Mikr. Anat., 85, 220-278.

HornitscheK (H.), I938. - Bau und Entwicklung der Locke des Karakulschafes. Kühn. Arch., 4\%, 6-I74.

Horio (M.), KONDO (T.), I953. - Crimping of wool fibres. Text. Res. J., 23, 373-387.

IBN HAUKAL, 978. - (Rapporté par d'AIGNEAUX, I928).

Iwanov (M. F.), Judin (B. M.), Brigis (O. N.), Poloschenzevoj (F. D.), Baranov (A. G.), Kononovor (A. P.), 1932. - Fourrure de 1'agneau Karakul. en russe. Institut moskovite d'élevage ovin à Sostava, $265 \mathrm{pp}$.

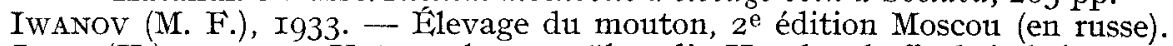
JAHN (K.), I923. - Untersuchungen ïber die Haarbeschaffenheit beim Karakulschaf. Dissertation. Halle.

JUdin (V.), I933. - Bases de la connaissance de la toison en relation avec la sélection des agneaux. en russe. Inst. Nat. de Rech. sur l'élevage du mouton (B. N. I. P. O.), Moscou.

JUdIN (V. M.), I937. - Problème de sélection dans l'élevage de Karakul (en russe). Probl. jivtn., 9, 66-78.

KASSENBECK (P.), I956. - Application de la microscopie électronique à l'étude de la structure fine des fibres de cellulose régénérée. Bull. I. T. F., 59, 7-2I.

KOEPPEL (K.), I9I9. - Das Karakulfellchen. Akklimatisation. Abband lung d. Farmwirtschafs gesellschaft für Südwestafrika, no 4.

Kononova (A. P.), I937. - Variation de la qualité des peaux Karakul pur et croisé durant les processus de manufacture (en russe). Usp. Zootech. Nauk., 4 (3), 3I-55.

LANGLET (J.), I936. - Einiges über Haut, Haar und Lockenbildung beim Karakulschaf. $Z$. Schafzucht, 17-18, 253-258.

Langlet (J. F.), Thomson (A. D.), Nel (J. A.), Scherz (E. R.), I947. - Bonitur-Anleitung des Karakul. Zuchtvereins für S.W. A. Verlag John Meinert, Windhoek, S. W. A.

LEveAu (M.), CeBE, (N) PARISOT (A.), I953.- Hétérogénéité de la fibre de laine et la réaction d'Allwörden. Bull. I. T. F. 42, 2-Io.

MARGolENA (L. A.), I954. - Sequence and growth of primary and secondary fibre follicles in karakul sheep. J animal Sci., 13, 765-780.

MAY (R. M.), r952. - La greffe. Ed. Gallimard, Paris, 299 pp.

MEHNERT (C.), I92r. - Erläuterung zum Boniturschema Karakuls. Mitteilungen der Farmwirtschaftsgesellschaft, 3.

MEIJERE (J. C. H. de), I894. - Uber die Haare der Säugetiere besonders über ihre Anordung. G. Morph. Jb, 21, 3I2-424.

Mercer (E. M.), I953. - The heterogeneity of the keratin fibres. Text. Res. $J ., 23,388-397$. 
Mercer (E. H.), GoldenN (R. L.), JeFiries (E. B.), I954. - Distribution of cystin in the cortex of wool. Text. Res. J., 24, 6I5-6I8.

Nathusius (W. von), I866. - Das Wollhaar des Schafes in histologischer und technischer Beziehung mit vergleichender Beriicksichtigung anderer Haar und Haut. Wiegant und Hempel, Berlin, 200 pp.

Nicov (Th.), I930. - Recherches sur les boucles des agneaux Karakul (en roumain). Bull. Minist. agric. Bucarest., 112, I44-219.

Nicov (Th.), I 934. - Étude sur le bouclage et la composition des boucles des agneaux de race Tzourcana grise de Roumanie. Ann. Inst. Nat. Zootech. Roumanie, 3, I75-20I.

Nikor JSKI (N. F.), I932. - Formation de la boucle chez la peau d'agneau Karakul (en russe). Chapli. Zoot. Exp. Sta., U. R. S. S., r, I5-46.

PFEIFER (E.), I929. - Untersuchungen ibler das histologische Zustandekommen der Lockung, mit besonderer Berücksichtigung des Karakullammes. Biol. Gener., 5, 239-266.

PFEIFER (E.), I953. - Karakul Atlas. Karakul breeders association of $S$. W. A., 25 I pp., Windhoek.

Rougeot (J.), I953. - La mesure du diamètre des brins de laine. Amn. Zootech., 4, 365-376.

ROUGEO'T (J.), I956. - Communications personnelles.

RUMEAU (J.), r 947. - Le mouton Karakul. Thèse méd. vét. Lyon, nº 7 .

RYDER (M. I.), I956. - The pre-natal development of follicles population in the Romney lamb. J. agric. Sci., 4\%, 6-IX.

Sanson (A.), I896. - Traité de Zootechnie, tome 5. Librairie agricole, Paris.

SCHINCKEL (P. G.), I955. - The pre-natal development of the skin follicle population in a strain of Merino sheep. Aust. J. agric. Res., 6, 68-76.

SHorT (B. F.), I955. - Development of the secondary follicle population in sheep. Aust. J. of agric. Res., 6, 62-67.

SPÖTTEL (W.), TäNZER (E.), I923. - Rassenanalitische Untersuchtungen an Schafen. Arch. Naturgesch., 89, I-242.

STICKER (A.), I887. - Uber die Entwicklung und den Bau des Wollhaares, nebst einem Anhang über das Wollfett. Landr'.Jb. 16, 625-657.

STOEHR (Ph.), I904. - Entwicklungsgeschichte des menschlichen Wollhaares. Arb. Anat. Inst. Wiesbaden, $n^{\circ}$ 7I, XXIII, I-66.

TÄNZER (E.), I928. - Haut und Haar beim Karakul in rassenanalytischem Vergleich. Kuhn. Arch., 18, I52-307.

TERENTJEVA (A. A.), I937. - Die Entwicklung der Wolldecke bei den Kirgisischen Fettsteisschafen. C. R. Acad. Sci. U. R. S. S. 16, I89-IgI.

Thompson (A. D.), I938. - Karakul Sheep. Verlag John Meinert, Windhoek, Afrique du Sud-Ouest.

ToLDT (K.), I9Ir. - Uber Hautzeichnung bei dichtbehaarten Säugetieren insbesondere bei Primaten. Zool. Jahrb. Abt. Systematik., 33.

VIGIER (P.), Ig04. - Mécanisme histologique de la frisure des productions pileuses. C. R. Assoc. Anat. 6, I76-I85.

Wahl (R. O.), I920. - Karakul sheep. J. Dept. agric. Union S. Africa, 1, 536-547.

Wildman (A. B.), I932. - Coat fibre development in some British sheep. Proc. zool. Soc. London, 2, 257-285.

Wil,dman (A. B.), CaR'TER (H. B.), I939. - Fibre follicle terminology in the mammalia. Nature, 144, $783-784$.

Wool industries research association, I948. - Detailed description of the projection methods for the measurement of fibre diameter to be used in the I948-9 experiments. I. W. T. O. Tech. Cttee. Proc., 2, 22-26.

Wool industries research association, I947. - The structure of animal fibres (A review of knowledge and of present work). Wool Ind. Res. Assn. Bull., 11, I9-23. 